\title{
An efficient approach based on selective partitioning for maximal frequent itemsets mining
}

\author{
ANITA BAI $^{1,2, *}$, MEERA DHABU $^{1}$, VIRAJ JAGTAP ${ }^{3}$ and PARAG S DESHPANDE ${ }^{1}$ \\ ${ }^{1}$ Department of Computer Science and Engineering, Visvesvaraya National Institute of Technology, \\ Nagpur 440010, India \\ ${ }^{2}$ Department of Computer Science and Engineering, Bharat Institute of Engineering and Technology, \\ Hyderabad 501510, India \\ ${ }^{3}$ Amazon, Hyderabad, India \\ e-mail: anita.bai@students.vnit.ac.in; anitabai@biet.ac.in; mmd@cse.vnit.ac.in; viraj1jagtap@yahoo.co.in; \\ psdeshpande@cse.vnit.ac.in
}

MS received 16 February 2017; revised 26 July 2018; accepted 16 May 2019

\begin{abstract}
We present a maximal frequent itemset (MFI) mining algorithm based on selective partitioning called SelPMiner. It makes use of a novel data format named Itemset-count tree-a compact and optimized representation in the form of partition that reduces memory requirement. It also does selective partitioning of the database, which reduces runtime to scan database. As the algorithm progressively searches for longer frequent itemsets in a depth-first manner, it creates new partitions with even smaller sizes having less dimensions and unique data instances, which results in faster support counting. SelPMiner uses a number of optimizations to prune the search space. We also prove upper bounds on the amount of memory consumed by these partitions. Experimental comparisons of the SelPMiner algorithm with popular existing fastest MFI mining algorithms on different types of datasets show significant speedup in computation time for many cases. SelPMiner works especially well when the minimum support is low and consumes less memory.
\end{abstract}

Keywords. Data mining; itemset-count tree; maximal frequent itemsets; partitions; transactional databases.

\section{Introduction}

Frequent itemsets mining (FIM) is one of the fundamental problems in data mining and has many applications such as association rule mining [1], query expansion [2], high utility mining [3, 4], occupancy-based frequent pattern (FP) mining [5], classification [6, 7], clustering [8], etc. Frawley and others studied knowledge discovery and defined it as 'the non-trivial extraction of useful information from data' [9]. Agrawal et al proposed association rules as a means of knowledge discovery and supportconfidence framework [10]. The problem of mining association rules was broken down into two parts of mining frequent itemsets in the database and of generating rules from these FPs [1]. In the proposed approach, we concentrate on the former one.

The problem of counting the number of maximal frequent itemsets (MFIs) for a given threshold is \#P-complete [11-13] and that of enumerating the set of MFIs is NP-hard. MFIs are orders of magnitude fewer in number than frequent itemsets and contain all the information contained in

*For correspondence

Published online: 17 July 2019 a set of frequent itemsets. The drawback of mining all frequent itemsets is that if there is a large frequent itemset with size $n$, then almost all $2^{n}$ candidate subsets of the items might be generated. However, since frequent itemsets are upward closed, it is sufficient to discover only all MFIs. A frequent itemset is called maximal if it has no superset that is frequent. Mining MFI from a large database requires novel mining algorithms as the existing algorithms are inefficient because of runtime and memory constraints. In many applications (especially in dense data) with long maximal FPs, enumerating all possible $2 m-2$ subsets of a ' $m$ '-length pattern ( $m$ can easily be 30 or 40 or longer) is computationally infeasible. Thus, there has been recent interest in mining maximal FPs in these 'hard' dense databases. Therefore, the design of an efficient algorithm would be governed by three issues: 1 . enumeration of all candidate itemsets, 2. counting supports of itemsets and 3 . making sure only MFIs are output.

A key challenge in MFI mining is to design effective and powerful search space pruning techniques to efficiently discover frequent items. To reduce the cost of multiple database scans and to efficiently remove impertinent items, we have proposed the MFIs mining algorithm based on selective partitioning, called SelPMiner. This algorithm 
recursively creates ordered partition, and merges transactions that are identical in each partition database using a linear time and space implementation.

\section{Contributions}

We propose SelPMiner, an efficient algorithm for enumerating the set of all MFIs. There are a number of innovative ideas employed in the development of SelPMiner, summarized as follows.

1. Increased compactability and optimized physical representation: We have proposed a modified compact data structure, viz, reduced transaction pattern list (RTPL) with Itemset-count tree, which stores only unique transactions of frequent items of the database in a bitmap while maintaining the count of its duplicates, which reduces runtime memory requirement while generating MFI. With RTPL representation, the memory requirement is dependent on the number of frequent items. Since the counts of duplicates are maintained, the memory requirement is also proportional to the MFI length and MFI count. The memory requirement is almost independent of the size of the original database. Moreover, the modified RTPL need not fit into memory, because proposed selective partitioning demands only relevant transactions of an item to be loaded into memory instead of complete RTPL representation of the database.

2. Cost-effective database scan: The proposed SelPMiner algorithm creates ordered partition for processing each itemset in which size of newly created partition reduces logarithmically. Moreover the searching in partition for determining MFI is done with a unique search technique as discussed in section 5.3, which provides $(\log N)$-time instead of $N$-time, where $N$ is the size of partition. Effect of partitioning approach on reduction of database scans is shown in table 10.

3. Horizontal and vertical reduction of a partition: The size of the partition is further reduced by proposed tail-count list structure as described in section 5.4a. According to our knowledge this is the first algorithm that does selective partitioning not only on data instance but also on dimensions for mining MFI.

4. Selective partitioning: Recursive partitioning reduces finite search cost, but at the same time it introduces overhead of creating partitions. However, the proposed SelPMiner algorithm creates partition selectively by determining the worst-case search cost. If the cost is less, only then the partition is created selectively as discussed in section 5.6.

5. Locality of reference: The searching is done in contiguous memory location, which introduces temporary locality of reference, which can be extracted by underlying memory cache management.
6. Efficient operations: SelPMiner does most of the operations using efficient AND/OR operators, which can be exploited by underlying hardware.

7. Efficient processing of high-dimensional very large database: Very large databases can be compressed and stored on disks in the form of RTPL representation using the proposed algorithm.

Summarizing, RTPL is used to increase compactability, for optimized physical representation and cost-effective intelligent selective partitioning. The large reduction in size of partition $(<<\log N)$ and reduction in cost of searching in the partition reduce the total runtime cost of search while generating MFI. Reduction in partition size also results in substantial reduction in runtime memory requirement.

Experimental comparisons of SelPMiner algorithm with popular existing fastest MFI mining algorithms FPMax* and PADS on a number of datasets with varying characteristics indicate that the proposed SelPMiner algorithm can be used to efficiently mine MFIs. The performance of SelPMiner with varying number of transactions in the modified RTPL and effects of partitioning strategy is presented in this paper. SelPMiner gives significant good results in terms of computation time and memory.

On the application side, max-patterns are used in mining maximal sequential patterns, web log mining [14], condensed representations of constrained FPs, summarizing association rules [15], combinatorial pattern discovery in biological discovery [16], mining borderline description, plagiarism detection, etc.

Rest of this paper is organized as follows. In section 3, we discuss background of the work. In section 4, we describe various aspects of the problem and build a platform to propose our algorithm and discuss representation of itemsets/transactions. In section 5, we develop our partition-based approach for problem decomposition and pattern search with faster support counting. Experimental results and comparisons are described in section 6 and we conclude in section 7 .

\section{Related work}

The problem of mining frequent itemsets was first introduced by Agrawal et al [10], who proposed the algorithm Apriori. Apriori uses a bottom-up approach. It uses hashtrees to store frequent itemsets and candidate frequent itemsets. For MFI mining, the key is an algorithmic principle. For instance, Apriori was the first to give good results. Then, FP-growth introduces a novel algorithmic principle associated with a data structure.

There has been much research on methods for generating MFIs efficiently [13, 17]. Zaki [13] introduced a depth-first search algorithm called Eclat, in which a database is vertically represented. Eclat involves a linked list approach to organize frequent itemsets. However, transaction IDs are 
stored in an array (the TID-array) with respect to each itemset, which requires more memory consumption. FPgrowth method has been proposed by Han et al [18]; in this approach the original database is stored using an FP-treebased data structure. Only two database scans are needed for the algorithm and no candidate generation is required. Results indicate that the FP-growth method is faster than Apriori. Nevertheless, since it enumerates all FPs it is impractical when pattern length is long.

The pattern-growth-based algorithm Afopt was proposed by Guimei Liu et al to mine closed itemsets and MFIs [19]. The basic operations in the pattern growth approach are counting frequent items and construction of new conditional databases [20]. It adaptively uses three different structures: arrays, Afopt-tree and buckets, to represent conditional databases according to the density of database. Grahne and Zhu [21] presented the FPmax algorithm for mining MFIs using the FP-tree structure. This approach traverses a search space in depth-first manner. In FPmax, a tree structure similar to the FP-tree is used for maximality testing. Takeaki Uno et al proposed LCM ver. 2 algorithm for finding frequent, closed and maximal itemsets. LCM algorithm and its variants are based on the backtracking algorithms, and use a technique for the frequency counting that is based on occurrence deliver and anytime database reduction [22].

Douglas et al [23] introduced Mafia algorithm for mining MFIs, which removes non-maximal sets using three pruning techniques. The first technique is the look-ahead pruning, which is used in the MaxMiner [24] method also. The second technique is to check if a new candidate itemset is a subset of an existing maximal set. The last technique checks if a superset of $\mathrm{X}$ is already considered frequent. Mafia uses vertical bitmap representation of data and projection of bitmaps to improve mining performance. GenMax proposed by Gouda and Zaki [25] uses an approach called progressive focusing to eliminate non-maximal itemsets. In this method it maintains a set of local MFIs and compares newly found FI itemsets with the small set of local MFIs. Mafia mines a superset of the MFI, and requires a postpruning step to eliminate non-maximal patterns, which takes too much time; in contrast, GenMax returns the exact MFIs. Mafia and GenMax use vertical database format, where TIDset for each item is available, which is the set of all transaction TIDs where they occur. The vertical methods spend much time in performing intersections on long item TIDsets or bit-vectors. GenMax gets around this problem using the horizontal format for computing frequent 2-itemsets (denoted as $F_{2}$ ), but it still has to spend time performing $\mathrm{O}\left(\left|F_{2}\right|\right)$ pairwise TIDset intersections. Mafia on the other hand performs $\mathrm{O}\left(\left|F_{1}\right|^{2}\right)$ intersections, where $F_{1}$ is the set of frequent items.

Grahne and Zhu [26] propose an algorithm that uses the FP-tree data structure in combination with the FP-array technique and incorporate various optimization techniques. These techniques are applied on the previous proposed algorithms, i.e., FPmax, FPgrowth and FPclose, and introduced FPmax*, FPgrowth* and Fpclose algorithms. FPmax* algorithm uses the MFI-tree data structure in combination with the FP-array technique. This algorithm works very well, especially when the dataset is sparse and very large. However, when a dataset is dense, the FP-trees become more compact. For each item in a compact FP-tree, the traversal is fairly rapid, while accumulating counts in the associated FP-array takes more time. Zeng et al proposed PADS method [27], which is a pattern-aware method. It uses the patterns already found to schedule its future search. The basic operations in the pattern growth approaches like Afopt and PADS are counting frequent items and new conditional databases construction. Therefore, the number of conditional databases constructed during the mining process and the mining cost of each individual conditional database have a direct effect on the performance of a pattern growth algorithm. The total number of conditional databases mainly depends on the order in which the search space is explored. The traversal cost and construction cost of a conditional database depend on the size, the representation format (tree-based or arraybased) and construction strategy (physical or pseudo) of the conditional database. These algorithms take more memory to store the conditional databases and require more time to explore the search space.

From this literature, we can conclude that plenty of algorithms have been proposed during the last decade for MFI mining, yet most of these algorithms are costly in terms of runtime and memory usage. The performance of mining algorithms mainly depends on used data structure and efficiency of generating and counting itemsets.

We solved these issues by exploring two ways of optimization. (i) Reducing cost of database scan by representing database using efficient data-structuremodified RTPL, which requires very less space to store input database. It stores only unique transactions of frequent items of the database in a bitmap while maintaining the count of its duplicates, which reduces runtime memory requirement while generating MFI. (ii) Enumerating less number of probable candidates using tailcount list and faster support counting with better pruning techniques.

\section{Preliminaries}

The problem of mining MFIs has received generous attention and this has resulted in the development of multitude of approaches to tackle it. It would be prohibitive to discuss all of them here and hence we discuss only those issues of the problem and alternative solutions that directly bear on the proposed solution. Table 1 summarizes the notations that will be used in this paper and their meaning.

The problem of mining maximal FPs can be formally stated as follows. Let $\mathcal{D}$ denote a database of transactions, 
Table 1. Notation and meaning.

\begin{tabular}{lc}
\hline Notation & Meaning \\
\hline$I$ & Set of items \\
$\mathcal{D}_{1}$ & Database \\
$\mathcal{D}_{2}$ & Partition database \\
threshold & Minimum support \\
$F$ & Set of all frequent itemsets \\
$F_{1}$ & Set of frequent 1 -itemsets \\
$k_{1}$ & Size of $F_{1},\left|F_{1}\right|$ \\
MFI & Set of maximal frequent itemsets \\
LMFI & Set of local maximal frequent itemsets \\
$R T P L$ & Reduced transaction pattern list \\
CE & Counting enumerator \\
PEP & Parent equivalence pruning \\
FHUT & Frequent head union tail \\
HUTMFI & Check head union tail in the MFI \\
\hline
\end{tabular}

where each transaction has a unique identifier $\left(T_{i}\right)$ and contains a set of items. The set of all TIDs is denoted as $T=\left\{T_{1}, T_{2}, \ldots, T_{n}\right\}$.

Definition 1 Let $I=\left\{i_{1}, i_{2}, i_{3}, \ldots, i_{k}\right\}$ be a set of items. Any subset of $I$ is called an itemset. An itemset with cardinality $k$ is called $k$-itemset.

Definition 2 The support of an itemset is defined as the number of transactions in which the itemset is present.

Definition 3 If the support of an itemset is greater than or equal to input threshold count, then the itemset is a frequent itemset (FI). We call the set of frequent 1-itemsets as $F_{1}$.

Definition 4 A frequent item $e$ is called an extension or child of a frequent itemset $X$ if $X \cup\{e\}$ is also frequent. The extension set of $X$ is the set of all extensions of $X$.

All frequent subsets of this itemset must be frequent. If a frequent itemset has cardinality $x$, then there are bound to be $2^{x}$ frequent itemsets that are subsets of the itemset [28]. Hence, an algorithm for mining frequent itemsets can generate a large number of FIs [28] to the knowledge gained. To solve this problem, one concentrates on the most generating FIs. One such measure of 'interestingness' is the length of a frequent itemset.

Definition 5 A frequent itemset is maximal if its extension set is empty.

Addition of any item to an MFI would result in an infrequent itemset. Given a user-specified support, our goal is to efficiently enumerate all patterns in MFI.

\subsection{Data representation}

4.1a Representation of itemsets: Let $|I|=k$. We can encode all possible itemsets under $I$ into $k$-bit binary numbers with each bit associated to an item in $I$. A set bit of such a binary number signifies the presence of an associated item in the itemset represented by that binary number.

Example 1 Consider $I=\{a, b, c, d\}$ and in the binary number $b_{0} b_{1} b_{2} b_{3}$, bits $b_{0}, b_{1}, b_{2}$ and $b_{3}$ represent items $a, b$, $c$ and $d$, respectively; then $(1010)_{2}$ would denote itemset $\{a, c\}$.

$4.1 \mathrm{~b}$ Database representation: The internal representation of database directly affects the support counting operation. A good representation should allow faster support counting and be space conservative. We discuss two basic representations as follows:

Horizontal: Horizontal representation stores database as a set of $(T I D, X)$ tuples, where TID is transaction identifier and $X$ is the list of items (itemset) in that transaction. Recall that itemsets can be encoded into binary numbers. A possible implementation could store each transaction as a $k$ bit binary number. However, notice that, after the first counting operation to determine $F_{1}$ (at root node in SETree), bits representing infrequent items would be unused. Hence, an efficient implementation first scans the database to enumerate set of frequent items $F_{1}$ and then stores the transactions in $k_{1}$-bit binary numbers (root node skips support counting). Table 2 presents an example database $\mathcal{D}_{1}$ and table 3 presents database $\mathcal{D}_{1}$ with infrequent itemsets removed, which is counted in first enumeration $F_{1}$ and sorted according to increasing order of support count of $F_{1}$ items on transactions. Table 4 presents horizontal representation of this sorted database.

Vertical: Vertical representation employs $\left(i, T_{i}\right)$ tuples, where $i$ is an item and $T_{i}$ is the list of transactions in which $i$ is present. This scheme simplifies support counting as $\operatorname{support}(i)=|T|$.

For an itemset $X=\left\{i_{1}, i_{2}, i_{5}\right\}$, the vertical representation can be calculated and stored as $\left(X,\left\{T_{1} \cap T_{2} \cap T_{5}\right\}\right)$. For conserving space, the transaction list could be stored as a bit sequence $b_{0} b_{1} \ldots b_{|\mathcal{D}|-1}$, with $b_{j}$ set to 1 if itemset is present in $j$ th transaction. Vertical representation of database $\mathcal{D}_{1}$ with infrequent itemsets removed is shown in table 5 .

Table 2. An example database $\mathcal{D}_{1}$.

\begin{tabular}{lc}
\hline TID & Item list \\
\hline 1 & $a, c, d, e, m$ \\
2 & $a, f, k$ \\
3 & $c, e, p$ \\
4 & $a, c, e, j, k, m, r$ \\
5 & $a, b, c, e, m$ \\
6 & $a, g, h$ \\
7 & $c, e$ \\
8 & $c, d$ \\
9 & $c, d, e, j, m$ \\
10 & $a, b, c, e, f, m$ \\
\hline
\end{tabular}


Table 3. Database $\mathcal{D}_{1}$ with infrequent itemsets removed.

\begin{tabular}{lc}
\hline TID & Item list \\
\hline 1 & $a, c, d, e, m$ \\
2 & $a$ \\
3 & $c, e$ \\
4 & $a, c, e, m$ \\
5 & $a, c, e, m$ \\
6 & $a$ \\
7 & $c, e$ \\
8 & $c, d$ \\
9 & $c, d, e, m$ \\
10 & $a, c, e, m$ \\
\hline
\end{tabular}

Table 4. Horizontal representation of database $\mathcal{D}_{1}$.

\begin{tabular}{lc}
\hline TID & $\begin{array}{c}\text { Representation } \\
\{\text { acdem }\}\end{array}$ \\
\hline 1 & 11111 \\
2 & 10000 \\
3 & 01010 \\
4 & 11011 \\
5 & 11011 \\
6 & 10000 \\
7 & 01010 \\
8 & 01100 \\
9 & 01111 \\
10 & 11011 \\
\hline
\end{tabular}

Table 5. Vertical representation of database $\mathcal{D}_{1}$.

\begin{tabular}{lc}
\hline Item & Representation \\
\hline$a$ & 1101110001 \\
$c$ & 1011101111 \\
$d$ & 1000000110 \\
$e$ & 1011101011 \\
$m$ & 1001100011 \\
\hline
\end{tabular}

\subsection{Approaching the problem}

We start with a naive approach to generate the set of MFI. As MFI $\subseteq$ FI, compute the power set of $I$, and check its supports to filter the frequent itemsets. This method explores $2^{k}-1$ candidate itemsets and hence it is impractical.

Nevertheless, the design of such an algorithm would be governed by three issues:

1. enumeration of all candidate itemsets;

2. counting supports of itemsets and

3. making sure only MFIs are output.

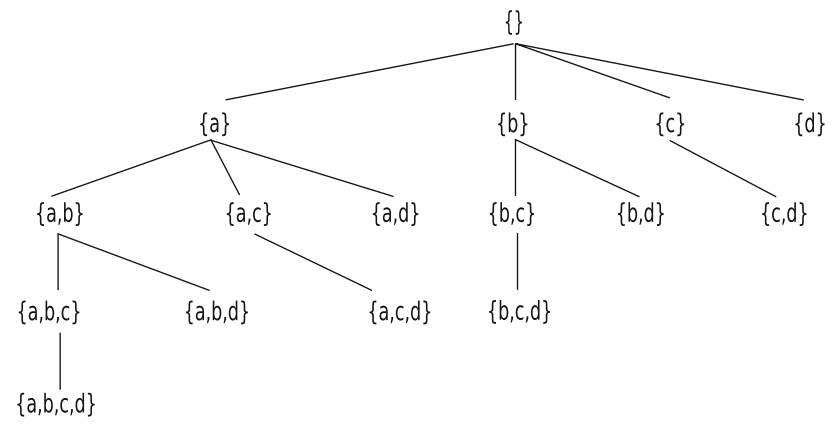

Figure 1. SE-Tree for a set of 4 elements.

\subsection{Enumeration of candidate itemsets}

Rymon [29] proposed an abstract 'Set Enumeration'-tree for enumerating sets for $I=\{a, b, c, d\}$; an SE-Tree-assisted enumeration of $P(I)$ is shown in figure 1. Consider a simple Counting Enumerator (CE) and an algorithm CEMiner using CE. CE simply counts binary numbers from 0 to $2^{k}-1$. Since each binary number represents an itemset, it indeed enumerates every possible candidate. For $I=\{a, b, c, d\}, \mathrm{CE}$ generates candidate itemsets in the following order: 0000, 0001, 0010, 0011, ..., 1110, 1111. $\mathrm{CE}$, though extremely simple, fails to capture the spirit of Apriori principle. ${ }^{1}$

\section{SelPMiner for efficient MFI mining}

The proposed SelPMiner algorithm uses a new data format named Itemset-count tree to partition the database. This is the innovation in this work. The algorithm progressively searches for longer supersets of an itemset using binary search and faster support counting with better pruning before recursing over a node. It creates new partitions with even smaller sizes, which results in faster support counting.

There are two main ingredients to reduce the search space while mining MFIs. The first is the set of techniques used to prune entire branches of the search space, and the second is the representation used to perform fast frequency computations.

\subsection{Reduced transaction pattern list (RTPL)}

Observe the horizontal representation of database in table 4. The horizontal representations of transactions 4, 5 and 10 and some others are equal. It is redundant to store these transactions separately. The duplicate transaction itemsets can be safely removed as long as we store one

\footnotetext{
${ }^{1}$ Apriori principle states that if an itemset is infrequent, all its supersets must also be infrequent [28].
} 
Table 6. RTPL.

\begin{tabular}{lllllc}
\hline \multicolumn{2}{l}{ Bit sequence } & & & \\
\hline$a$ & $c$ & $d$ & $e$ & $m$ & Count \\
\hline 0 & 1 & 0 & 1 & 0 & 2 \\
0 & 1 & 1 & 0 & 0 & 1 \\
0 & 1 & 1 & 1 & 1 & 1 \\
1 & 0 & 0 & 0 & 0 & 2 \\
1 & 1 & 0 & 1 & 1 & 3 \\
1 & 1 & 1 & 1 & 1 & 1 \\
\hline
\end{tabular}

copy of the itemset and the number of its duplicates using proposed RTPL approach [30] as follows:

1. remove each item $i$ where support $_{i}<$ threshold;

2. keep only unique transaction itemsets and remove duplicate transaction itemsets while maintaining the number of its duplicates.

Table 6 shows the RTPL for the database in table 4 with bit sequences sorted by their binary values. To construct the RTPL of any database $\mathcal{D}_{1}$, the database is first scanned for frequent items. After associating every item to a bit position in the binary number, the database is scanned for second time to convert transactions to their horizontal representations. The itemsets are kept in sorted order to facilitate faster duplicate counting.

$I^{*}$ This function is used for construction of RTPL

Input: $D$, a transactional database.

Output: Reduced transactions pattern list, RTPL.

1 foreach transaction $T$ in $\mathcal{D}$ do

2 foreach item $i$ in $T$ do

$I \leftarrow I \cup\{i\}$; support $_{i} \leftarrow$ support $_{i}+1$;

5 foreach item $i$ in $I$ do

$6 \quad$ if support $_{i} \geq$ threshold then

7 | $F_{1} \leftarrow F_{1} \cup\{i\}$;

8 foreach transaction $T$ in $\mathcal{D}$ do

9 itemset $\leftarrow \phi$;

foreach item $i$ in $T$ do

if $i \in F_{1}$ then

itemset $\leftarrow$ itemset $\cup\{i\}$;

if itemset present in $R T P L$ then

| RTPL.count $\leftarrow R T P L$.count +1 ; else

RT PL.count $\leftarrow 1$;

17 return RTPL ;

Algorithm 1: constructRTPL $(\mathcal{D})$

Algorithm 1 demonstrates RTPL construction. This representation not only compresses the database but also quickens support counting. As we will see now, RTPL is an instance of partition that can be implemented as Itemsetcount tree. Algorithm 2 shows how extensions/children for a frequent itemset can be found. The support of individual extensions is incremented by counts of the itemset in RTPL if that extension item is present in that particular RTPL.

$I^{*}$ This function is used to enumerate child nodes and apply PEP technique to count support of frequent items */

Input: $R T P L$, reduced transactions pattern list; $\mathrm{N}$, processing Node.

Output: The child node list of N, children.

1 foreach itemset $T$ in $R T P L$ do

$2 \quad$ if $N$.itemset $\subseteq T$ then

$3 \quad \mid$ foreach item $i$ in N.candidates do

$$
\text { if } i \in T \text { then }
$$

| support $_{i} \leftarrow$ support $_{i}+R T P L_{T}$.count;

6 foreach item $i$ in candidates do

\begin{tabular}{l|l}
7 & if support $_{i} \geq$ threshold then
\end{tabular}

\begin{tabular}{l|l|l}
8 & if support $_{i}=N$. support then
\end{tabular}

$9 \quad$ N.itemset $\leftarrow$ N.itemset $\cup\{i\} ; / *$ PEP */

$10 \quad$ else

$11 \quad \mid$ children $\leftarrow$ children $\cup\{i\}$;

12 sort children in increasing order of their supports; 13 return children;

Algorithm 2: enumerateChildren $(R T P L$, Node $\mathrm{N})$

\subsection{Partitions}

The problem of mining MFIs, as defined in section 4, requires enumeration of all MFIs whose support is no less than a given threshold value. Since all the MFIs must be enumerated, clearly the computation cost must be proportional to at least the number of all MFIs. On the other hand, an algorithm may not be able to enumerate all MFIs efficiently as the number of MFIs may be exponential in the worst case. In other words, the number of MFIs in database $\mathcal{D}_{1}$ is exponential of the size of $\mathcal{D}_{1}$. This suggests that if the size of $\mathcal{D}_{1}$ reduces, MFI can be efficiently extracted. Here, we realize the need of partitioning-based approach to reduce the size of database $\mathcal{D}_{1}$. Hence, we propose to recursively reduce the size of database $\mathcal{D}_{1}$ using Itemset-count trees as partitions with the help of a height-balanced tree, which enumerates MFI efficiently. This approach enumerates longer extension set $E$ of all frequent itemsets during recursive partitioning.

Definition 6 For an itemset $X$, the set of relevant transactions $\mathcal{R}_{X}$ is defined as

$$
\mathcal{R}_{X}=\{T: T \in \mathcal{D}, X \subseteq T\} .
$$

$\mathcal{R}_{X}$ lists the transactions of $\mathcal{D}$ that have $X$ in them. Trivially

$$
\operatorname{support}(X)=\left|\mathcal{R}_{X}\right| .
$$

Table 7 shows $\mathcal{R}_{X}$ where $X=\left\{e_{1}\right\}$. 
Table 7. Example database $\mathcal{D}_{2}: \mathcal{P}_{X=\left\{e_{1}\right\}}$.

\begin{tabular}{lcccccc}
\hline \multicolumn{2}{l}{ Bit sequence } & & & & \\
\hline$e_{1}$ & $e_{2}$ & $e_{3}$ & $e_{4}$ & $e_{5}$ & $e_{6}$ & Count \\
\hline$\ldots .$. & & & & & & $\ldots$ \\
1 & 0 & 0 & 0 & 0 & 1 & $c_{1}$ \\
1 & 0 & 0 & 0 & 1 & 0 & $c_{2}$ \\
1 & 0 & 1 & 0 & 1 & 1 & $c_{3}$ \\
1 & 0 & 1 & 1 & 1 & 0 & $c_{4}$ \\
1 & 0 & 1 & 1 & 1 & 1 & $c_{5}$ \\
1 & 1 & 0 & 1 & 0 & 1 & $c_{6}$ \\
1 & 1 & 0 & 1 & 1 & 0 & $c_{7}$ \\
1 & 1 & 1 & 1 & 1 & 1 & $c_{8}$ \\
\hline
\end{tabular}

Definition 7 For an itemset $X$, with set of extensions $Y$, the set of relevant transactions $\mathcal{R}_{X, Y}$ is defined as

$$
\mathcal{R}_{X, Y}=\left\{Z: A \in \mathcal{R}_{X}, Z=A \cap Y\right\}
$$

$\mathcal{R}_{X, Y}$ denotes the set of transactions that are relevant to $X$ but with items not present in $Y$ discarded.

Example 2 Table 7 shows a portion of RTPL of database with $k_{1}=6$ bits for the first frequent itemset $X=\left\{e_{1}\right\}$; the tail count of candidate items of itemset $X$ is computed as shown in table 8. From table 8 , we can see that $e_{2}$ is infrequent with itemset $X$. Hence, extension set of $X$ is $Y=\left\{e_{3}, e_{4}, e_{5}, e_{6}\right\}$. Since $X \cap Y=\phi$, the bits of items in $X$ are also discarded for further investigation.

Definition 8 The function of isolated support count of itemset $A$ in $\mathcal{R}_{X, Y}$ is

$$
\psi(A, X, Y)=|\{T: T \in \mathcal{D}, X \cup A=T \cap(X \cup Y)\}|
$$

where $A \in \mathcal{R}_{X, Y}$. The duplicate counts in RTPL can be thought of as $\psi$ operated on corresponding bit sequences.

Definition 9 The Partition $^{2}$ of $X$ over $Y, \mathcal{P}_{X, Y}$, is defined as set of tuples $(R, c)$ where $R \in \mathcal{R}_{X, Y}$ and $c=\psi(R, X, Y)$.

Example $3 \mathcal{P}_{X, Y}$ is depicted in table 9 for $X=\left\{e_{1}\right\}$ and $Y=\left\{e_{3}, e_{4}, e_{5}, e_{6}\right\}$. Finally, $\mathcal{P}_{\phi, F_{1}}$ represents the RTPL of complete database $\mathcal{D}_{1}$ as shown in table 6 .

Recall that the depth-first traversal of SE-Tree dictates early investigation of longer supersets of a frequent itemset.

Example 4 Horizontal and vertical reduction of a partition: In table 7, the rows of RTPL that are relevant to item 100000 (i.e., item $e_{1}$ ) are at the bottom because of the sorted order of the itemsets. An MFI mining algorithm using this list is currently processing itemset 100000 . Assume that the algorithm finds that 010000 (i.e., item $e_{2}$ )

\footnotetext{
${ }^{2}$ Partitions presented in this document should not be confused with the ones used in Partition algorithm [31]. The Partition algorithm just divides the dataset into smaller chunks to reduce I/O cost [31].
}

Table 8. Candidates support counting for partition $\mathcal{P}_{X, Y}$.

\begin{tabular}{lc}
\hline Candidates & Tail count \\
\hline$e_{2}$ & $c_{6}+c_{7}+c_{8}<$ threshold \\
$e_{3}$ & $c_{3}+c_{4}+c_{5}+c_{8}>$ threshold \\
$e_{4}$ & $c_{4}+c_{5}+c_{6}+c_{7}+c_{8}>$ threshold \\
$e_{5}$ & $c_{2}+c_{3}+c_{4}+c_{5}+c_{7}+c_{8}>$ threshold \\
$e_{6}$ & $c_{1}+c_{3}+c_{5}+c_{6}+c_{8}>$ threshold \\
\hline
\end{tabular}

Table 9. $\mathcal{P}_{X, Y}$ or $\mathcal{P}_{100000,001111}$.

\begin{tabular}{ccccccc}
\hline \multicolumn{2}{l}{ Bit sequence } & & & & \\
\cline { 1 - 1 } & $e_{2}$ & $e_{3}$ & $e_{4}$ & $e_{5}$ & $e_{6}$ & Count \\
\hline 0 & 0 & 0 & 0 & 0 & 1 & $c_{1}$ \\
0 & 0 & 0 & 0 & 1 & 0 & $c_{2}$ \\
0 & 0 & 0 & 1 & 0 & 1 & $c_{6}$ \\
0 & 0 & 0 & 1 & 1 & 0 & $c_{7}$ \\
0 & 0 & 1 & 0 & 1 & 1 & $c_{3}$ \\
0 & 0 & 1 & 1 & 1 & 0 & $c_{4}$ \\
0 & 0 & 1 & 1 & 1 & 1 & $c_{5}+c_{8}$
\end{tabular}

is not an extension of 100000 (that is 110000 is infrequent as $c_{6}+c_{7}+c_{8}<$ threshold), while others are. The traversal is now about to recurse over 101000. Any subsequent counting operations will never consider the second bit.

A shorter table representation that could be used to count support of supersets of itemset 101000 is shown in table 9 . It is obtained by clearing bits not set in itemset 001111 . This is similar to the situation where we removed infrequent items from horizontal representation because they would be unused. This leads to the revelation that RTPL can be constructed on any itemset of our choice by selecting only those rows/transactions that are relevant to the chosen itemset and preserving only those bits that are extensions of the itemset.

The partitions $\mathcal{R}_{X, Y}$ can be implemented with the help of a height-balanced tree. The tuples are stored in key-value format, with itemsets being the keys and the $\psi$ counts being their respective values. This structure is referred to as Itemset-count tree. This allows simple duplicate counting. For brevity, we refer to Itemset-count trees as partitions throughout this paper. The benefit of using partitioning method is discussed in sections 6.4 and 6.6.

Compact and optimized representation in the form of a partition reduces the time required for database scan, which can be seen in table 10. For Connect dataset, the original database of 67,557 transactions reduces to 5,663 transactions, which is the average size of the partition. Average size of partition is smaller by an order of 12 than that of the original database. Similarly, we can see the reduction by an order of 8, 12, 12, 12 and 4 for Webdocs, Accidents, Pumsb*, Pumsb and Chess datasets, respectively, for $10 \%$ minimum support. For 5\% minimum support, we can see 
Table 10. Average size of partitions.

\begin{tabular}{|c|c|c|c|c|c|c|}
\hline Dataset & $\# T$ & $\# \mathbf{I}$ & \#FI & \#MFI & APL & ASP \\
\hline \multicolumn{7}{|c|}{ Support $=10 \%$} \\
\hline Webdocs & $16,92,082$ & $52,67,656$ & 262 & 10,717 & 6.91 & 199,237 \\
\hline Connect & 67,557 & 129 & 73 & 130,986 & 21.12 & 5663 \\
\hline Accidents & 340,183 & 468 & 77 & 104,287 & 11.37 & 49,345 \\
\hline Pumsb* & 49,046 & 2088 & 110 & 16,437 & 14.29 & 4837 \\
\hline Pumsb & 49,046 & 2113 & 135 & 16,013 & 15.54 & 5,428 \\
\hline Chess & 3196 & 76 & 61 & $23,39,525$ & 15.63 & 924 \\
\hline \multicolumn{7}{|c|}{ Support $=5 \%$} \\
\hline Webdocs & $16,92,082$ & $52,67,656$ & 683 & 32,145 & 8.34 & 179,381 \\
\hline Connect & 67,557 & 129 & 86 & 413,053 & 22.72 & 5581 \\
\hline Accidents & 340,183 & 468 & 107 & 620,400 & 12.38 & 42,643 \\
\hline Pumsb* & 49,046 & 2088 & 147 & 98,140 & 16.5 & 4332 \\
\hline Pumsb & 49,046 & 2113 & 172 & 123,763 & 17.45 & 4928 \\
\hline Chess & 3196 & 76 & 66 & $57,84,232$ & 17.48 & 863 \\
\hline
\end{tabular}

$\# \mathrm{~T}=$ number of transactions, $\# \mathrm{I}=$ number of items, \#FI = number of frequent items $\left(F_{1}\right)$,

\#MFI = number of maximal frequent itemsets, APL = average pattern length and ASP $=$ average size of partitions (bits).

the reduction by an order of $9,12,4,8,12$ and 10 for Webdocs, Connect, Chess, Accidents, Pumsb* and Pumsb datasets, respectively.

FPmax* [26] uses FP-array data structure to store database, and SelPminer uses Itemset-count tree data structure as discussed in section 5.1. Itemset-count tree uses bit sequences to encode the items in transactions. By reordering the itemsets according to the bit values, similar itemsets sharing common prefix subsequences are grouped together. Though FPMax* uses FP-array structure, which efficiently extracts the MFI-tree, it takes more time than algorithms that follow encoding itemsets/transactions using bit sequences.

To deal with big data, many approaches have been proposed to parallelize most of the existing algorithms and some new approaches like [32-34] have also been proposed. These methods deal with two challenges, space complexity and time complexity, to mine FPs from big data. They usually adopt the following two approaches: (1) distributing the computing workload while restoring the data in a shared memory environment and (2) apply the Map-reduce framework to partition both the computing workload and the data. SelPMiner with new data format named partition can deal with these challenges efficiently for distributed computing environment.

\subsection{Faster support counting}

Algorithm 3 scans every tuple in RTPL. This can be improved by utilizing the sorted order of partition's tuples. Due to the nature of binary numbers in sorted order, bit values $(b)$ of adjacent numbers usually huddle together. For a particular bit position $i$, a section of numbers with $b_{i}=1$ is followed by a section of numbers with $b_{i}=0$ and vice versa. For example, in figure 2, for the third bit from left, i.e., $b_{2}$, first five values are 0 , followed by nine 1 's, then by four 0 's. That is, contiguous portions of relevant itemsets are followed by contiguous irrelevant parts. The irrelevant parts can be hopped over.

Example 5 Figure 2 shows a portion of a partition. Here, positions of binary numbers are indicated by bits. For

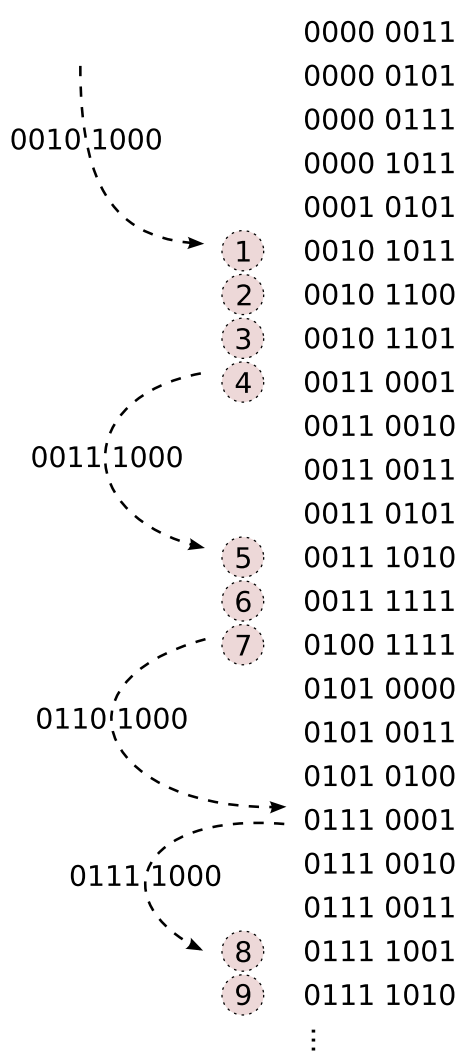

Figure 2. Counting 00101000. 
example, $X=00101000=\left(b_{1} b_{2} b_{3} b_{4} b_{5} b_{6} b_{7} b_{8}\right)$, where bit $b_{5}$ represents 1 . The algorithm needs to find extensions of $X$ $=00101000$ and hence needs to locate its supersets. The least binary number that is the superset of $X$ is obviously the itemset itself. We use the binary search of itemset $X$ over the RTPL list. The binary search is modified to return a number greater than or equal to its argument. Hence, it starts search from beginning of RTPL list and returns 0010 1011 , the closest number in the partition to $X$. After operating on (1), the algorithm checks if the next sequential number is a superset of (1), which it is, and the algorithm continuously finds supersets of $X$. The algorithm operates on (2) and (3). However, (4) (i.e., 00110001 ), say $C$ is not a superset of $X$. Now, we calculate the next possible superset of $X$ using (4). The rightmost bit in $C=$ (4) $=00110001$ turned 0 , but 1 in $X$ is set to 1 (i.e., bit $b_{5}$ ); hence, updated value will be $C=00111001$ and bit positions in $C$ right of $b_{5}\left(b_{6}\right.$ to $\left.b_{8}\right)$ are cleared to get $C^{\prime}=00111000$. Then $C^{\prime}$ is bitwise-ORed with $X$ to get the possible immediate superset 0011 1000. This is then searched to get (5).

$/^{*}$ This function is used for finding next superset of itemset quickly */

Input: $\mathcal{P}_{X, Y}$, partition on itemset X; Y, set of extensions of $\mathrm{X} ; \mathrm{X}$, an itemset.

Output: Next superset of $X, T$.

$1 T \leftarrow$ current;

2 while $T$ is not the last itemset in $P_{X, Y}$ do

$3 \quad T \leftarrow$ sequential successor of $\mathrm{T}$ in $\mathcal{P}_{X, Y}$;

if itemset $\subseteq T$ then return $\mathrm{T}$;

else

next $\leftarrow$ compute next smallest superset;

$T \leftarrow \operatorname{binaryS} \operatorname{earch}($ next $)$;

9 return null;

Algorithm 3: getNextSuperset $\left(\mathcal{P}_{X, Y}\right.$, itemset, current)

SelPminer maintains local as well as global MFI-list as an ordered bit sequence list for maintaining already discovered MFIs. Algorithm 6 implements binary search as discussed earlier over MFI-list for finding any superset of newly discovered MFI. FPMax* efficiently extracts the MFI-tree but takes more time to count supports. SelPMiner makes full use of the pruning power of the minimum support threshold to cut down search space dramatically.

\subsection{Recursive partitioning}

So far we have discussed only the viability of $\mathcal{P}_{X, Y}$ with any itemset $X$ and extension set $Y$ as a means of faster support counting. Now we consider a possible algorithm using partitions (Algorithm 7). RecMiner first follows Algorithm
1 to construct $\mathcal{P}_{\phi, F_{1}}$. At the root of SE-Tree, it recurses over the first frequent 1 -itemset $\in F_{1}$, say $A$. It then enumerates extension set $E=\left\{e_{0}, e_{1}, \ldots\right\}$ of $A=\{\phi\}$. RecMiner then constructs smaller partition $\mathcal{P}_{A, E}$ (Algorithm 4) and recurses over frequent itemset $A \cup\left\{e_{0}\right\}$. After returning from the recurse over $A \cup\left\{e_{0}\right\}$, RecMiner will never use data contained in the bit for $e_{0}$ in $\mathcal{P}_{A, E}$ (bit $e_{0}$ is still important in $\left.\mathcal{P}_{\phi, F_{1}}\right)$. RecMiner then compresses $\mathcal{P}_{A, E}$ to $\mathcal{P}_{A, E-\left\{e_{0}\right\}}$ (Algorithm 5) and recurses over $A \cup\left\{e_{1}\right\}$. By the time it processes the last child extension, the partition will contain one or two tuples. Most likely RecMiner will hit a prune by Algorithm 6 on one of the extensions, in which case RecMiner destroys the partition and returns. It now compresses $\mathcal{P}_{\phi, F_{1}}$ to $\mathcal{P}_{\phi, F_{1}-A}$.

$/ *$ This function is used for creation of new partition selectively from parent partition

Input: $\mathcal{P}_{X, Y}$, parent partition on itemset $\mathrm{X} ; X^{\prime}$, an itemset; $Y^{\prime}$, extension list of $X^{\prime}$.

Output: New partition from parent partition $\mathcal{P}_{X, Y}$, $\mathcal{P}_{X^{\prime}, Y^{\prime}}$

1 foreach superset $T$ of $X^{\prime}$ in $\mathcal{P}_{X, Y}$ do /* calls Alg.

3

$2 \quad T^{\prime} \leftarrow T \cap Y^{\prime} ;$ count $\leftarrow\left(\mathcal{P}_{X, Y}\right)_{T}$;

if $T^{\prime}$ in $\mathcal{P}_{X^{\prime}, Y^{\prime}}$ then

else

$\left(\mathcal{P}_{X^{\prime}, Y^{\prime}}\right)_{T^{\prime}} \leftarrow$ count

8 return $\mathcal{P}_{X^{\prime}, Y^{\prime}}$;

Algorithm 4: constructPartition $\left(\mathcal{P}_{X, Y}, X^{\prime}, Y^{\prime}\right)$

/* This function is used for compressing partition

Input: $\mathcal{P}_{X, Y}$, current partition on itemset X; $i$, item.

Output: $\mathcal{P}_{X^{\prime}, Y^{\prime}}$, compressed partition without item $i$.

1 foreach superset $T$ of $\{i\}$ in $\mathcal{P}_{X, Y}$ do

$2 \mid T^{\prime} \leftarrow T-\{i\}$;

$3 \quad$ count $\leftarrow\left(\mathcal{P}_{X, Y}\right)_{T}$;

if $T^{\prime}$ in $\mathcal{P}_{X, Y}$ then

$\left(\mathcal{P}_{X, Y}\right)_{T^{\prime}} \leftarrow\left(\mathcal{P}_{X, Y}\right)_{T^{\prime}}+$ count;

else

$\mid\left(\mathcal{P}_{X, Y}\right)_{T^{\prime}} \leftarrow$ count

remove $\left(\mathcal{P}_{X, Y}\right)_{T}$;

9 return $\mathcal{P}_{X^{\prime}, Y^{\prime}}$

Algorithm 5: compressPartition $\left(\mathcal{P}_{X, Y}, i\right)$

5.4a Faster support counting with recursive partitions: $\mathcal{P}_{A, E-\left\{e_{0}\right\}}$ is obtained from $\mathcal{P}_{A, E}$ in $\mathrm{O}\left(\left|P_{A, E}\right|\right)$ time by removing all transactions not including $e_{0}$ based on the equality $\mathcal{P}_{A, E-\left\{e_{0}\right\}}=\mathcal{P}_{A, E} \bigcap \mathcal{P}_{A, E-\left\{e_{0}\right\}}$. Thus the total 
computation for all $e \in E$ takes $|E|$ scans and $\mathrm{O}\left(\left|\mathcal{P}_{A, E}\right||E|\right)$ time.

Instead, for all $e \in E$, we maintain tail-count list, $T\left[e_{i}\right]=\left(\left|P_{A, E-e_{i}}\right|\right) . \quad$ Count $\left(\left|P_{A, E}\right|\right)=\sum_{i=1}^{n} R T P L_{i}$.count, where $R T P L_{i} \in \mathcal{P}_{A, E}$ simultaneously by scanning the RTPL in $\mathcal{P}_{A, E}$ at once as follows: we initialize $T[e]=\phi$ for all $e \in E$. For each $R T P L \in \mathcal{P}_{A, E}$ and for all $e \in E$, we compute $T[e]$. If $\operatorname{count}\left(\left|P_{A, E}\right|\right)=\operatorname{count}\left(\left|P_{A, E-Z}\right|\right)$, where $Z$ is an itemset and $Z \subseteq E$, then $\left(\left|P_{A, E}\right|\right)=\left(\left|P_{A, E-Z}\right|\right)$. For item $e_{i} \notin Z$ and $T\left[e_{i}\right]>$ threshold, RecMiner then compresses $\mathcal{P}_{A, E}$ to $\mathcal{P}_{A, E-Z}$ (Algorithm 5) and recurses over $A \cup\{Z\}$.

\section{/* This function is used to prune the search space by using pruning techniques}

Input: N, node/current processing itemset; MFI, maximal frequent itemsets list.

Output: Updated local maximal frequent itemsets list, LMFIs.

1 Longest $\leftarrow N$.itemset $\cup N$.candidates;

$2 L \leftarrow M F I$.current;

3 while $L$ is not the last itemset in MFI-list do

$L \leftarrow$ sequential successor of $L$ in MFI-list;

if Longest $\subseteq L$ then return true; $\quad / *$ HUTMFI */ break;

else

next $\leftarrow$ compute next smallest superset in

MFI-list;

$L \leftarrow \operatorname{binaryS} \operatorname{earch}($ next $)$;

11 return null;

12 foreach superset $T$ of Longest in N.P do

$13 \mid$ support $\leftarrow$ support $+(N . \mathcal{P})_{T}$;

14 if support $\geq$ threshold then

$15 \mid N . L M F I \leftarrow N^{\prime} . L M F I \cup$ Longest;

16 return true; $/ *$ FHUT */

17 else

18 return false;

19 return LMFI

Algorithm 6: FHUTorHUTMFI(Node N, MFI)

For example, we have a partition $\mathcal{P}_{X, E}$ with any itemset $X$ and extension set $E=\{f, b, c, d\}$. Algorithm 1 constructs $\mathcal{P}_{\phi, F_{1}}=\mathcal{P}_{\phi,\{f, b, c, d\}}$. RecMiner first constructs smaller partition for itemset $X=\{f\}, \mathcal{P}_{X, E}=\mathcal{P}_{f, E-\{f\}}$. We maintain tail-count list to calculate count of all items $e \in E$ and merge itemset $X$ with the item $e \in E$, which has the same count, i.e., the updated $X=\{f, b\}$, if we found count $\left(\left|P_{X, E}\right|\right)=\operatorname{count}\left(\left|P_{f, E-\{f\}}\right|\right)=\operatorname{count}(b)$, where $\operatorname{count}(b)$ $\in P_{f, E-\{f\}}$. After returning from the recurse over $\{f\}$, RecMiner will never use data contained in the bit for $\{f\}$ in $\mathcal{P}_{b, E}$. RecMiner then compresses $\mathcal{P}_{\phi, F_{1}}$ to $\mathcal{P}_{\phi, F_{1}-\{f\}}$ and recurses over $X=\{b\}$ at root.

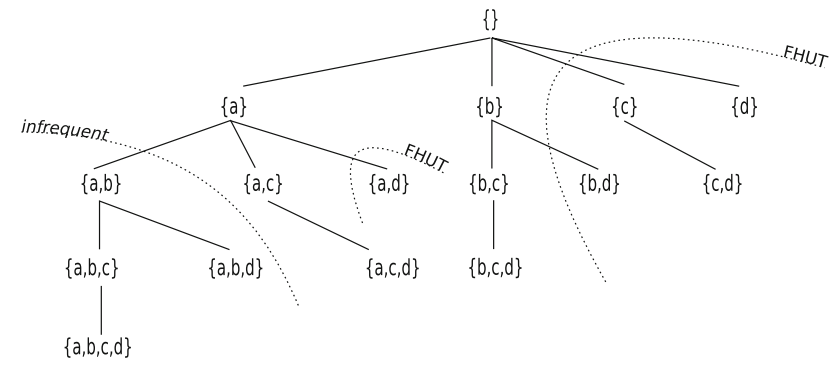

Figure 3. FHUT prunes.

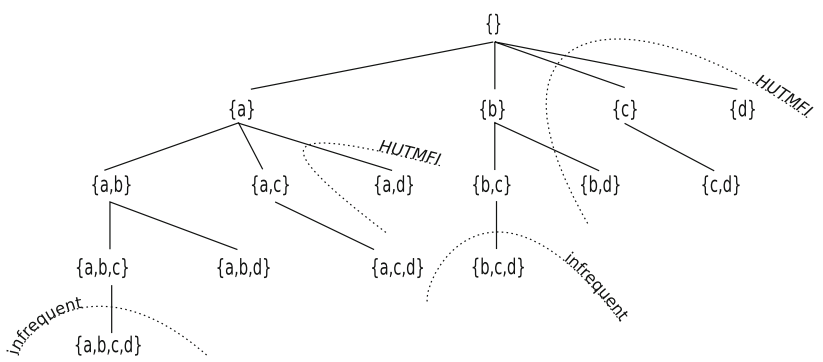

Figure 4. HUTMFI prunes.

\subsection{Search space reduction}

5.5a Frequent head union tail $(F H U T)^{3}$ : Let $A$ be a candidate FI and $C$ be its set of candidate extensions. If the longest possible superset of $A$, i.e. $(A \cup C)$, is discovered to be frequent, then there is no need to explore any subsets of $A \cup C$ and thus the entire subtree rooted at node $A$ can be pruned out. In figure $3,\{c\}$ and $\{d\}$ are the only candidate extensions to $\{a\}$. The longest possible superset of $\{a\}$ is $\{a, c, d\}$; it is found to be frequent and hence other children of $\{a\}$ are never explored. Similarly, the longest possible superset of $\{b\}$ is $\{b, c, d\}$; it is found to be frequent; thus $\{b, d\},\{c\}$ and $\{d\}$ can be pruned out; these cuts are shown by FHUT cut in figure 3 . This technique was employed in MaxMiner [24] and Mafia [23].

5.5b Check head union tail in MFI (HUTMFI) ${ }^{3}$ : If the longest possible superset of a candidate FI is a subset of an MFI, then the subtree rooted at the node corresponding to that itemset can be pruned away [23, 25]. In figure 4 , since one of the longest superset of node $\{a\}$ is $\{a, c, d\}$, which is found to be a subset of MFI, $\{a, d\}$ is pruned because $\{a, d\} \subseteq\{a, c, d\}$. Similarly, the other longest superset of node $\{a\}$ is $\{a, b, d\}$, which is found to be frequent; then $\{b, d\}$ is pruned because $\{b, d\} \subseteq\{a, b, d\}$. Similarly, $\{c\}$ and $\{d\}$ are pruned. These cuts are shown by the HUTMFI cut in figure 4.

5.5c Parent equivalence pruning and reordering: We use parent equivalence pruning (PEP) and reordering to cut many branches of the tree. Rearrange the children of each

\footnotetext{
${ }^{3}$ As observed by Gouda and Zaki [25], the names FHUT and HUTMFI were used by Burdick et al [23] in Mafia.
} 
node according to their support counts, allowing larger Apriori and HUTMFI prunes. This technique was employed in Mafia [23] and CHARM [35].

\section{$/ *$ RecMiner function is used to create} partitions recursively */

Input: $\mathrm{N}$, node (processing itemset); LMFI, old local maximal frequent itemsets list.

Output: Updated local MFIs, LMFIs.

\section{1 if $N$ is root then}

2 children $\leftarrow F_{1}$;

3 else

4 children $\leftarrow$ enumerateChildren(N.P, N.candidates);

5 if children $=\phi$ then

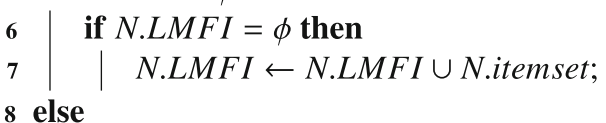

Algorithm 7: RecMiner(Node N, LMFI)

\section{Faster PEP with $\mathcal{P}_{A, E}$}

We maintain the tail-count list, which computes the count for each $e \in E$ simultaneously by scanning the $\mathcal{P}_{A, E}$ at once. Hence SelPMiner algorithm requires $\mathrm{O}\left(\left|P_{A, E}\right|\right)$ time for PEP.

5.5d Better pruning with recursive partition: The FHUT with reordering as mentioned in the previous section needs to recurse down to the actual longest frequent itemset. RecMiner instead prunes away at the source of the longest FI. Figure 5 shows the contrast between RecMiner's cuts and cuts shown in figures 3 and 4.

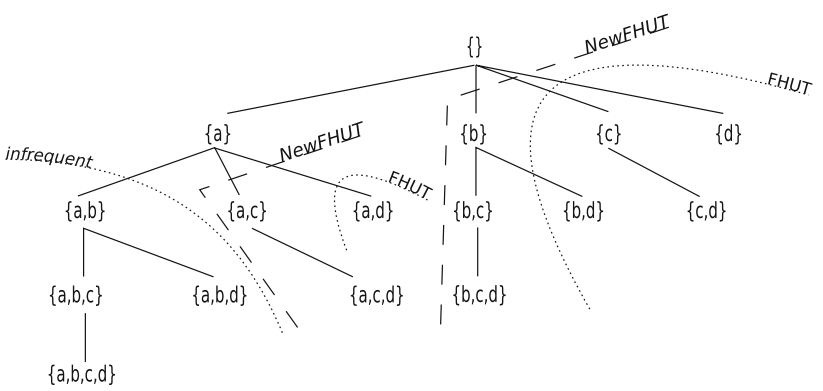

Figure 5. Better pruning.
RecMiner finds out that $\{b, c, d\}$ is frequent before recursing over $\{b\}$, while alternative algorithms need to traverse down to $\{b, c, d\}$. RecMiner is able to do so because partitions allow faster counting of any given itemset. It queries for occurrences of $\{b, c, d\}$ at null node (after having processed $\{a\}$ ) and finds it to be frequent. These cuts are shown by NewFHUT cut in figure 5 . Approaches like Mafia store data in vertical format; hence they would have to process transaction list of $\{b\},\{c\}$ and $\{d\}$ at null node to achieve the same effect, which would be expensive.

\subsection{Selective partitioning}

Algorithm 8 shows the process of recursive creation of selective partitions. In the worst case, the recursion tree can be $\mathrm{O}(n)$ deep if a partition is created for each item explored. Hence the number of partitions held can be $\mathrm{O}(n)$. To limit this usage we must create partitions judiciously. We propose to create a new partition only if the size of the new partition is half of that of the current partition. To this end, we propose a strategy to find an upper bound on the number of tuples the new partition may have. We modify Algorithm 2 for support counting to gather some statistics. While recording the support of extensions, we also record the number of tuples in which they are present.

Suppose, a parent partition $\mathcal{P}$ has $|\mathcal{P}|$ number of tuples. Let, for itemset $X$, the set of extensions be $E$ with $y$ items and $N$ be the number of $X$ 's relevant itemsets in $\mathcal{P}$. Extensions $e_{1}, e_{2}, \ldots, e_{y}$ are present in $N_{1}, N_{2}, \ldots, N_{y}$ number of relevant tuples, respectively, with $N_{1}, \ldots, N_{y}$ arranged in decreasing order. The obvious upper bound on the size of new partition $\left|\mathcal{P}_{X, Y}\right|$ would be $2^{y}$. If $2^{y} \leq|\mathcal{P}| / 2$, we stop and decide to create $\mathcal{P}_{X, E}$. If not, we try to further tighten the bound. For binary numbers between 0 and $2^{y}$, a bit position is ' 1 ' only for half of the numbers in that range, that is $2^{y-1}$ numbers. $e_{1}$ is present in $N_{1}$ tuples. The new partition in the worst case can have $2^{y-1}$ number of tuples with $e_{1}$ in them. The new bound will be equal to $2^{y-1}$ plus the number of tuples without $e_{1}$ in them. If $2^{y-1}+(N-$ $\left.N_{1}\right) \leq|\mathcal{P}| / 2$ we create the partition. Else we proceed further. The number of tuples with both $e_{1}$ and $e_{2}$ in them for new partition would be bounded by $2^{y-2}$. In the present partition, $e_{1}$ and $e_{2}$ can be present in $N_{1}+N_{2}-N$ tuples. Hence, the $N_{1}+N_{2}-N$ tuples would be reduced to $2^{y-2}$ tuples. Thus, the bound will be $\left(N-\left(N_{1}+N_{2}-N\right)\right.$ $\left.+2^{y-2}\right)$. We continue to calculate the bound till it is decreasing. If it increases, we stop. If the tightest bound is less than $|\mathcal{P}| / 2$, we create a new partition. Else we decide not to create partition $\mathcal{P}_{X, E}$ but try to find bound on $\mathcal{P}_{X, E-\left\{e_{1}\right\}}$. We find the extension set $E^{\prime}$ for which the bound is acceptable. This operation adds little overhead as it runs in $\mathrm{O}(y)$ time. 
/* SelPMiner function is used for recursive creation of selective partitions

Input: $\mathcal{D}$, a transactional database; threshold, a minimum support threshold.

Output: The set of maximal frequent itemsets, MFIs.

$/ *$ For first function call:

$/ * F_{1} \leftarrow$ Frequent itemsets list;

$/ *$ N.LMFI $\leftarrow$ Local maximal frequent itemsets list;

$/ *$ MFI $\leftarrow$ Maximal frequent itemsets list;

1 Set $N$.candidates $\leftarrow I, N$.itemset $\leftarrow\{\phi\}$,

$N$.support $\leftarrow|\mathcal{D}|, N . L M F I \leftarrow\{\phi\}, M F I \leftarrow\{\phi\}$,

$N . \mathcal{P} \leftarrow \mathcal{P}_{\phi, F_{1}} ;$

$2 R T P L \leftarrow$ constructRT PL $(\mathcal{D})$;

3 if $N$ is root then

$4 \mid$ children $\leftarrow F_{1}$;

5 else

6 children, pItem $\leftarrow$ enumerateChildren(N.P, N.candidates);

7 if children $=\phi$ then

$8 \quad$ if $N . L M F I=\phi$ then

$9 \mid N . L M F I \leftarrow N . L M F I \cup N . i t e m s e t$;

10 else

$11 \mid X \leftarrow N$.itemset; $Y \leftarrow$ children;

$\mathcal{P} \leftarrow N . \mathcal{P}$;

foreach $i$ in children do

construct itemset, candidates, LMFI, support of $N^{\prime}$;

$N^{\prime} . \mathcal{P} \leftarrow \mathcal{P}$

if FHUTorHUTMFI $\left(N^{\prime}, L M F I\right)$ then

break loop;

if $i=$ pItem then

$\mathcal{P} \leftarrow \mathcal{P}_{X, Y} \leftarrow$

constructPartition $(N . \mathcal{P}, X, Y)$;

$\operatorname{RecMiner}\left(N^{\prime}, \mathrm{LMFI}\right)$;

$\mathrm{MFI} \leftarrow M F I \cup \mathrm{LMFI}$;

if new partition was created then

$\mathcal{P} \leftarrow \operatorname{compress} \operatorname{Partition}(\mathcal{P}, i)$;

else

$Y \leftarrow Y-\{i\}$

$\left.25\right|_{26}$ return MFIs ;

Algorithm 8: SelPMiner(D, threshold)

At any given time, selective partitioning will have partitions that are half the size of their own parent partitions. Hence the number of tuples held by all partitions will be bounded by $2 \times \mid$ RTPL $\mid$.

\subsection{Handling high-dimensional database}

With the rapid development of technological progress, the amount of data with large number of attributes stored in databases increases rapidly. These databases can be viewed as high-dimensional databases, since the attributes correspond to the dimensions of the dataset. Many real-life applications rely on very large databases consisting of millions of data objects with several hundreds to a few thousands of dimensions. Mining MFI from high-dimensional data requires novel mining algorithm as the existing algorithms are incompetent because of memory constraints.

One of the challenges for high-dimenstional database is the curse of dimensionality. The complexity of many existing data mining algorithms is exponential with respect to the number of dimensions. With increasing dimensionality, these algorithms soon become computationally intractable for very low minimum support and therefore inapplicable in many real applications. Because the dimensionality of database is usually big, and without pruning the huge search space, one has to generate a very large set of candidate itemsets for checking, which requires huge memory.

SelPMiner solves these challenges as follows:

- One approach to minimize memory requirement is to divide high-dimensional database into a number of disjoint partitions and loading a single partition in memory at a time for mining.

- Size of each partition depends on the number of items/ dimensions and number of data instances in that partition.

- Horizontal reduction of a partition results in reduced number of data instances while vertical reduction results in reducing dimensions. SelPMiner does efficient mining of high-dimensional database as it partitions database horizontally as well as vertically.

- If a partition is very large and cannot fit into memory then its memory requirement is reduced by creating a new partition from existing partition, making algorithm robust for any size/dimension of data.

\subsection{Correctness and completeness}

\section{Theorem 1 (Correctness) SelPMiner enumerates} $\forall \mathrm{MFI} \subseteq$ FI.

Proof Let node $N=\bigcup_{i=1}^{m} N_{i}$ represent frequent $m$-can didate itemsets. Node $N^{\prime}=\operatorname{tail}(N)$ represents a child-node of $N$, where $N^{\prime} \in \mathrm{FI}\left(\mathcal{P}_{\mathrm{X}, \mathrm{Y}}\right)$ with respect to the item count of $T(N)$. Therefore, $N^{\prime}=\bigcup_{i=1}^{n} \mathrm{FI}_{i}(\operatorname{tail}(N))$. Thus, the complete set of the MFI can be generated by $N$.

SelPMiner correctly identifies all and only MFIs, since its search is based on a complete SE-Tree search space. The only branches that are pruned are those that either do not have sufficient support, or those that are subsumed by another itemset based on the properties of PEP based on selective partitioning as outlined in lines (7-9) of Algorithm 2. Finally, SelPMiner eliminates any non-maximal itemset that might be generated by performing faster 
superset checking before inserting locally discovered MFI in the set of global MFI.

Theorem 2 (Completeness) SelPMiner returns global $\mathrm{MFI}=\bigcup_{i=1}^{n} \mathrm{MFI}_{i}$ and $\mathrm{MFI} \subseteq \mathrm{FI}$.

Proof Let global list MFI $=\bigcup_{i=1}^{n} \mathrm{LMFI}_{i}$ consist of all local $n$-MFI list. $M^{\prime}=\left\{\mathrm{LMFI}_{1} \cap \mathrm{LMFI}_{2} \cap \cdots \cap \mathrm{LMFI}_{n}\right\}$ is the correct intersection of MFI if and only if $M^{\prime} \neq \forall \mathrm{LMFI}_{i}$.

SelPMiner correctly returns all local LMFIs, which combine as a global MFI list. In the depth-first traversal of SE-Tree, an LMFI holds only the superset MFIs of the FI currently being investigated. A new $\mathrm{LMFI}_{i+1}$ is created with MFIs if $I_{i+1} \bigcup Y_{i+1}$ is found to be frequent, since $I_{i+1} \cup Y_{i+1}$ is a superset of $I_{i+1}$. When a superset check is to be made, it suffices to check |LMFI|. If $|\mathrm{LMFI}| !=\{\phi\}$ it indicates the presence of supersets in MFI. The concept of mining MFI using LMFI is presented in line 15 of Algorithm 6, line 7 of Algorithm 7 and line 20 of Algorithm 8.

\section{Experimental results}

We use Mafia, GenMax, Afopt (max), FPmax* and PADS as the current state-of-the-art method for MFI mining and show how SelPMiner compares against it. All our experiments were performed on a Intel core i3 CPU with $4 \mathrm{~GB}$ RAM. The operating system was Ubuntu 14.04 LTS using Linux 3.19. All codes of the SelPMiner were written in $\mathrm{C}++$ and compiled using gcc version 4.8.2. The implementations of Mafia, Afopt and FPmax* were obtained from FIMI repository, ${ }^{4}$ and GenMax ${ }^{5}$ and PADS $^{6}$ were also obtained for comparisons. Both time and peak memory consumption of all algorithms running on each dataset were recorded by the 'time' and 'memusage' command, respectively.

\subsection{Datasets}

Several datasets are taken from Frequent Itemset Mining Dataset Repository. ${ }^{4}$ Chainstore, a large dataset, was obtained from NU-Mine Bench 2.0 [36]. All datasets with some characteristics such as number of transactions, items and average transaction length are shown in table 11.

Satisfactory results for small datasets indicate that if we perform the entire process on massive/large datasets then it is possible to give the same performance for maximal frequent itemset mining in terms of completeness and correctness. SelPMiner gives the desired degree of speedup, as we can see in section 6.5 for large datasets. Sampling

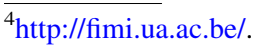

${ }^{5}$ http://www.cs.rpi.edu/ zaki/www-new/pmwiki.php/Software/ Software\#toc 24

${ }^{6}$ http://www.cs.sfu.ca/ jpei/Software/PADS.zip.
}

Table 11. Datasets.

\begin{tabular}{|c|c|c|c|c|}
\hline Dataset & $\# \mathrm{~T}$ & $\# \mathrm{I}$ & ATL & Type \\
\hline Webdocs & $16,92,082$ & $52,67,656$ & 71.47 & $\begin{array}{c}\text { Sparse very } \\
\text { large }\end{array}$ \\
\hline $\begin{array}{l}\text { BMS- } \\
\quad \text { WebView-2 }\end{array}$ & 77,512 & 3340 & 4.62 & Sparse \\
\hline $\begin{array}{l}\text { BMS- } \\
\text { WebView-1 }\end{array}$ & 59,602 & 497 & 2.51 & Sparse \\
\hline Retail & 88,162 & 16,469 & 10.3 & Sparse \\
\hline Chainstore & $11,12,949$ & 46,086 & 7.2 & Sparse large \\
\hline Accidents & 340,183 & 468 & 33.8 & Dense large \\
\hline Connect & 67,557 & 129 & 43 & Dense \\
\hline Pumsb & 49,046 & 2113 & 74 & Dense \\
\hline Pumsb* & 49,046 & 2088 & 50.5 & Dense \\
\hline Chess & 3196 & 76 & 37 & Dense \\
\hline
\end{tabular}

$\# \mathrm{~T}=$ number of transactions, $\# \mathrm{I}=$ number of items and $\mathrm{ATL}=$ average transaction length.

technique on massive databases would be very interesting. As suggested by Chakaravarthy et al [37] the accuracy obtained on the sample will remain the same if we work with massive datasets, and they give a comprehensive explanation for the well-known empirical successes of sampling for association rule mining.

\subsection{The runtime}

Figure 6 shows the run time performance of the six algorithms on the real datasets, viz, Accidents, Pumsb, Chess, Connect and Pumsb*, which are dense datasets, and figure 7 shows on Webdocs, Retail, Chainstore, BMS-WebView-1 and BMS-WebView-2, which are sparse datasets. While conducting experiments to compare the six different algorithms, we observe that the performance can vary depending on the dataset characteristics. The datasets Chess and Pumsb are characterized by a symmetric distribution of the maximal FPs and these patterns are relatively short (average length is 11 for chess and 10 for Pumsb) [25]. Figure 6(a) shows the experimental result on Chess. Chess has very less number of items, so that SelPMiner prunes many branches of the tree using recursive partitioning with PEP and returns the actual longest frequent itemset. The pattern-aware dynamic search approach of PADS takes more time than SelPMiner. FPMax* uses FP-array data structure with MFI-tree to store database and works very well, especially when the dataset is sparse and very large. However, when a dataset is dense, the FP-trees become more compact. For each item in a compact FP-tree, the traversal is fairly rapid, while accumulating counts in the associated FP-array can take more time as can be seen in figure 6(a)-(d). For lower minimum support, performance of PADS matches that of Afopt and GenMax shown in figure 6(a). Figure 6(b) shows experimental results on Pumsb. For lower support, we find that SelPMiner is faster 


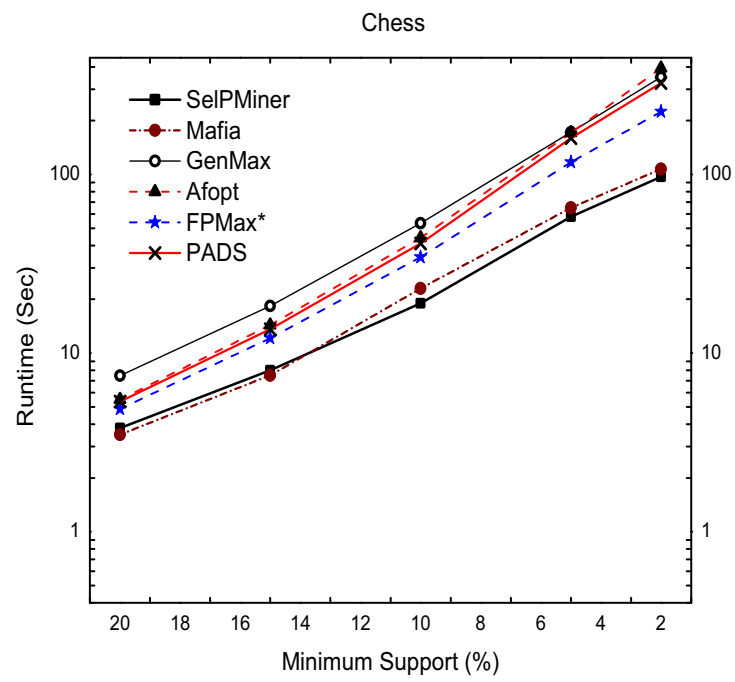

(a)

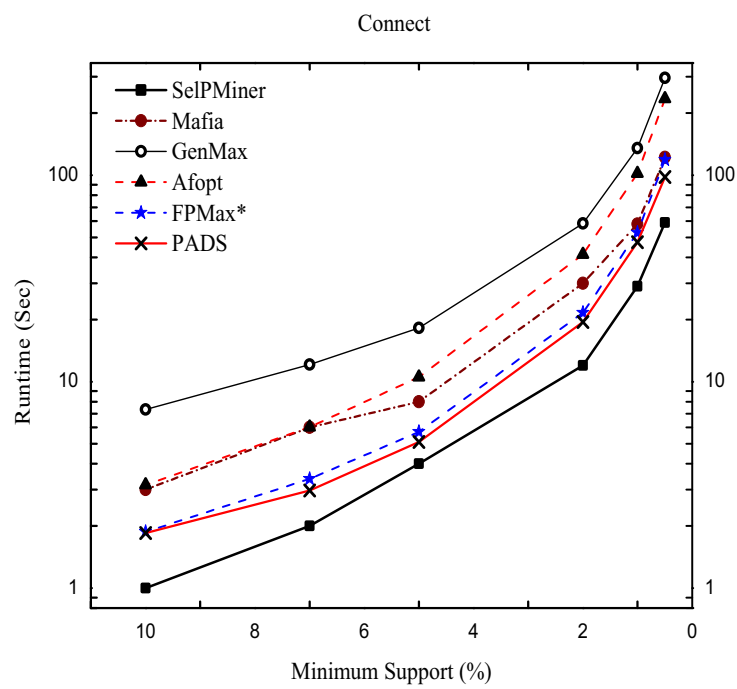

(c)

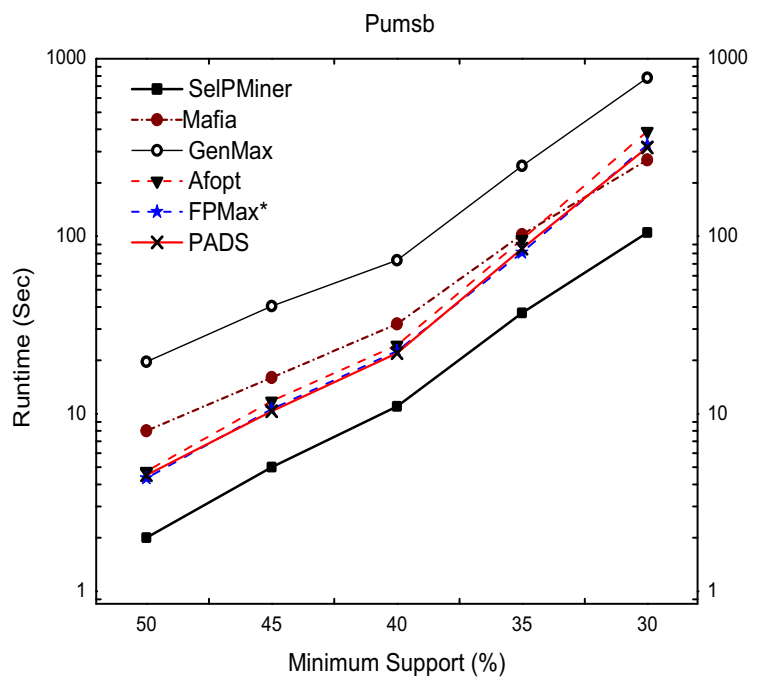

(b)

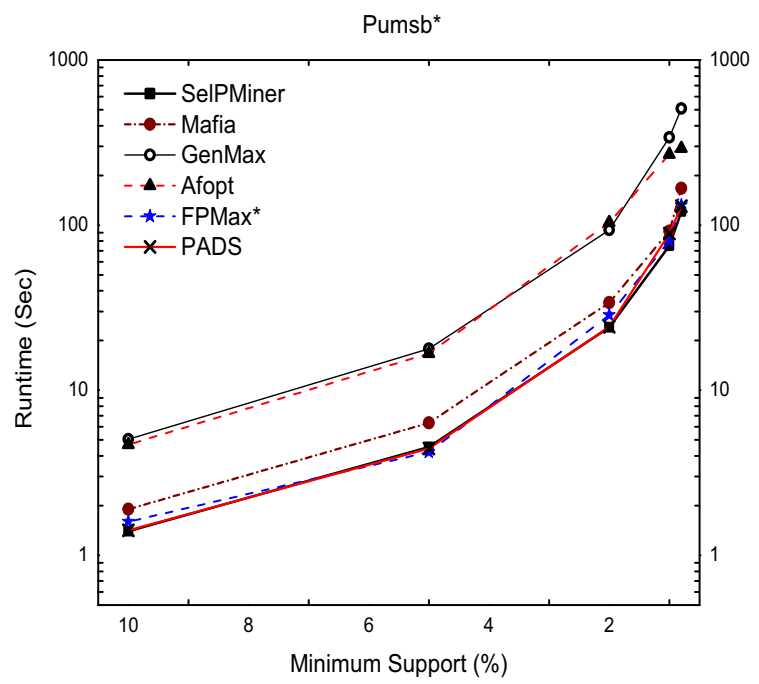

(d)

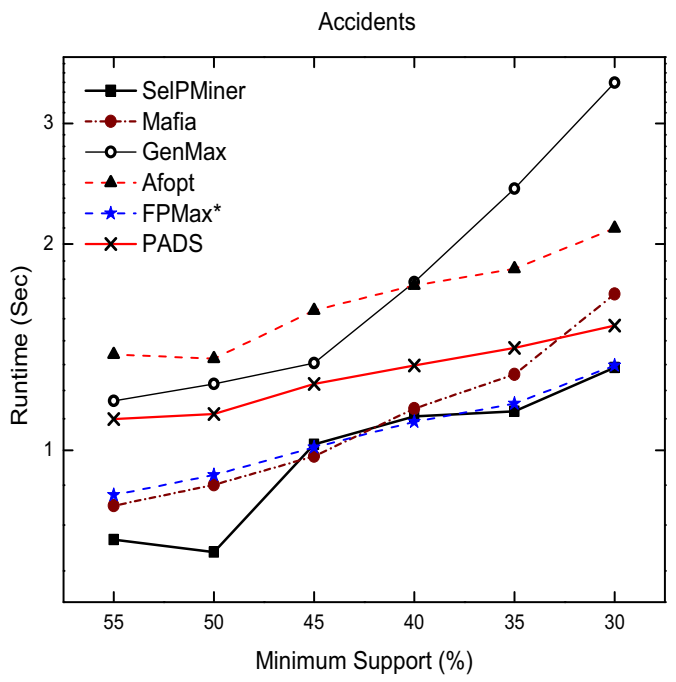

(e)

Figure 6. Runtime of mining MFIs on dense datasets. 


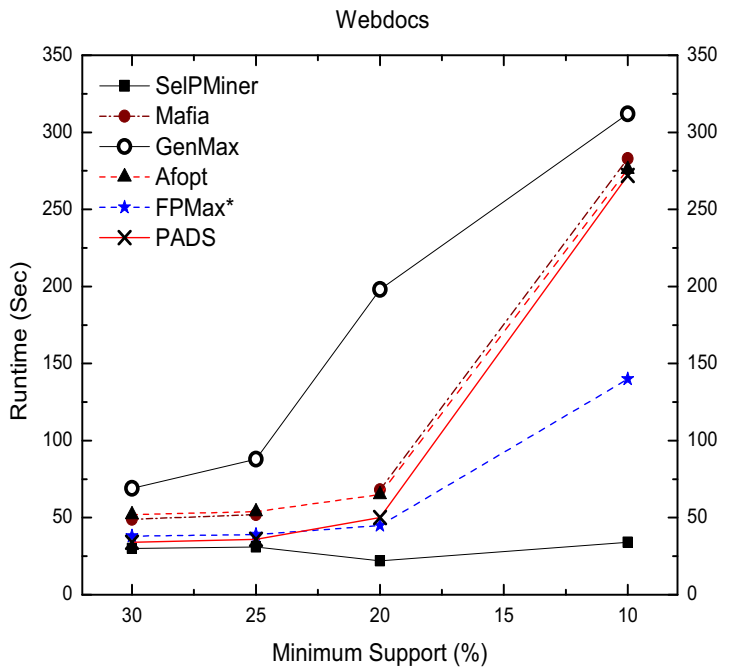

(a)

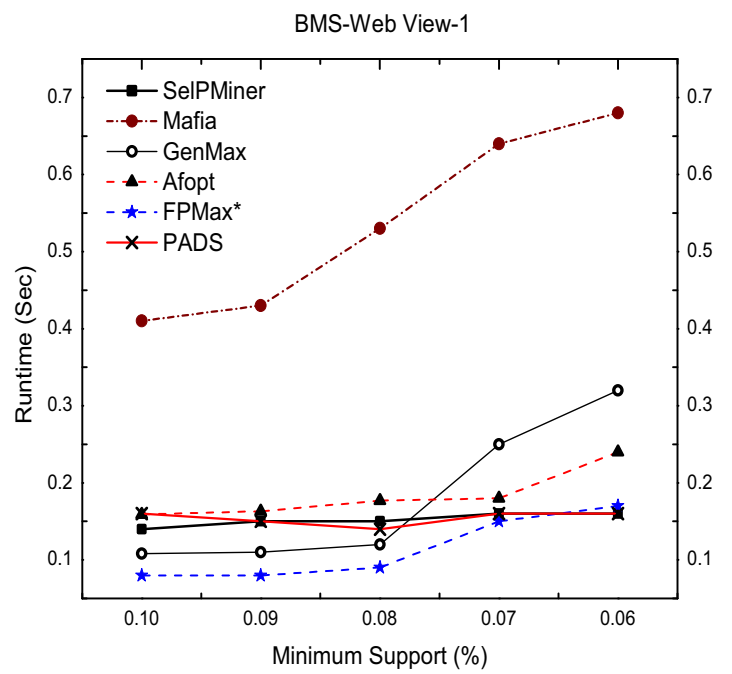

(c)

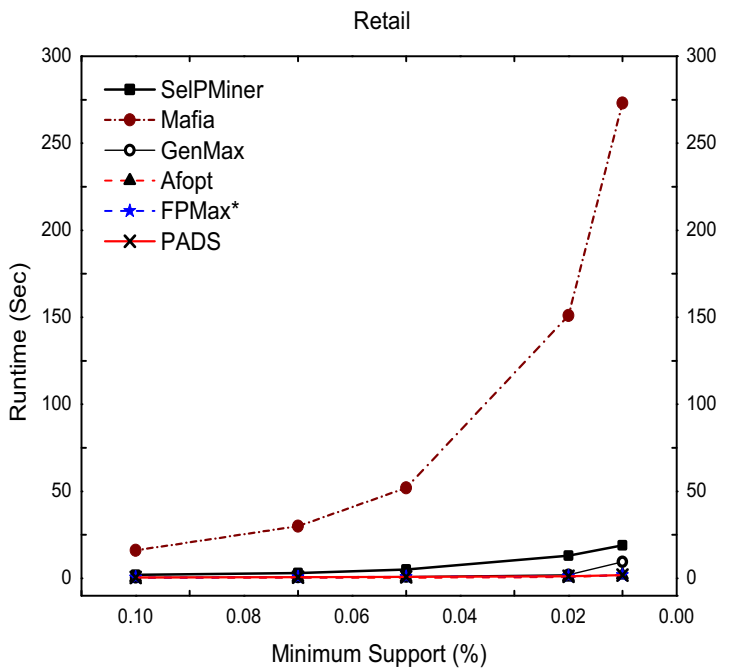

(b)

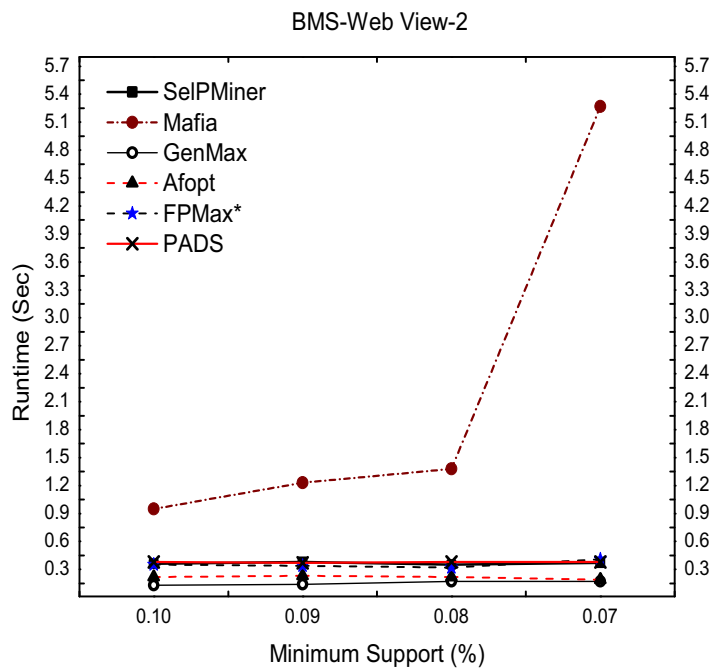

(d)

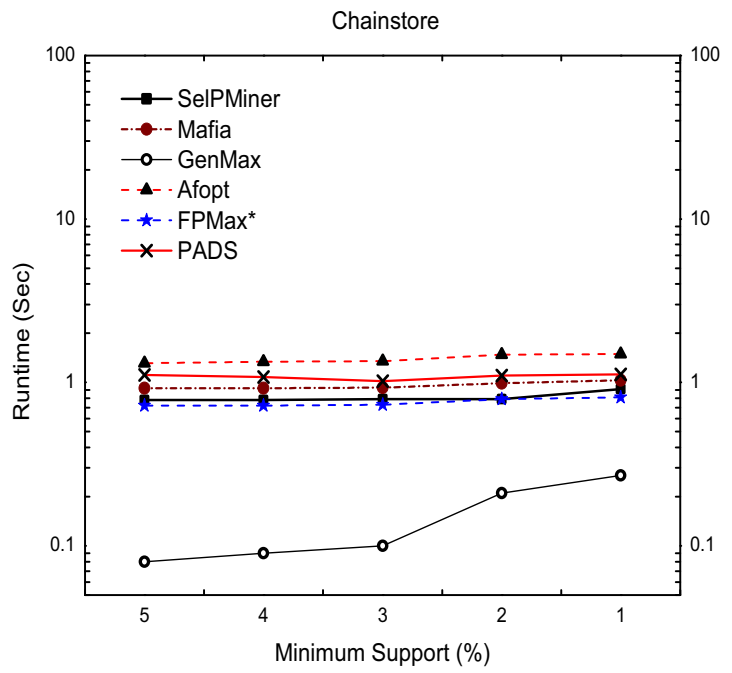

(e)

Figure 7. Runtime of mining MFIs on sparse datasets. 
by a factor of 10 than GenMax, 2.5 times faster than Afopt, FPMax* and PADS, and 3 times faster than Mafia. GenMax shows exponential growth for lower support values on Pumsb.

The datasets Connect and Pumsb* are characterized by a left-skewed distribution of the maximal FPs, i.e., there is a relatively gradual increase with a sharp drop in the number of maximal patterns [25]. Figure 6(c) shows experimental results on Connect. For higher as well as lower support, we find that SelPMiner outperforms all other algorithms. The reason is most likely due to the benefits of recursive partitioning, i.e., reducing dataset size, which results in efficient counting. As we can see from this figure, the proposed approach SelPMiner outperforms the currently fastest maxpattern mining algorithms FPMax* and PADS in a clear margin for low and higher support. Figure 6(c) clearly shows that PADS outperforms FPMax* on all supports. The lower the support threshold, the larger the difference in runtime. With a smaller support threshold, more patterns and longer patterns are qualified as FPs. This trend suggests that PADS is more scalable than FPMax* on mining a large number of long patterns.

Figure 6(d) shows experimental results on Pumsb*. SelPMiner exhibits performance comparable to that of the currently fastest max-pattern mining algorithm FPMax*, but it is faster than Mafia, GenMax and Afopt algorithms. The performances of GenMax and Afopt are almost same but five times slower than that of SelPMiner. GenMax increases exponentially with very low minimum support. This increase happens due to storage of very large MFItrees, i.e., a large number of MFIs in memory.

Figure 6(e) shows experimental results on Accidents dataset. Accidents dataset has very large itemsets and does not lend itself to faster FHUT pruning. On Accidents, we find that SelPMiner is the fastest compared with all algorithms for high value of support. This is possible as SelPMiner can do fast FHUT pruning because of selective partitioning, which results in fast support count with the help of tail-count list in reduced partition. FPmax* uses FParray structure, which efficiently extracts the MFI-tree but takes more time than those of algorithms that follow bitmap representation of database, i.e., Mafia and SelPMiner. SelPMiner and Mafia are able to count supports faster than FPMax* because of this database representation as can be seen in figure 6(e). However, Mafia does not return the exact MFIs; rather, it returns a superset of maximal patterns. For low value of support, SelPMiner exhibits the best performance, having a slight edge over FPMax*, but it is still 1.5 times faster than GenMax and 2 times faster than Afopt algorithms.

Figure 7(a) shows experimental results on Webdocs dataset. The results indicate that SelPMiner performs well against all other algorithms on high-dimensional sparse very large dataset for low minimum support and is 10 times, 8 times, 7.2 times and 7 times faster than GenMax, Mafia, PADS and Afopt, respectively. Figure 7(b) shows experimental results on Retail dataset. Mafia shows superexponential growth and thus becomes impractical for datasets having long maximal patterns at lower supports. Retail dataset leads to a large number of transactions; Mafia's vertical representation leads to its poor performance as can be seen in figure 7(b). PADS, FPMax* and GenMax exhibit the best performance, having a slight edge over SelPMiner. SelPMiner almost overlaps FPMax* and PADS for high support.

Mafia has the worst performance on BMS-WebView-1 and BMS-WebView-2 datasets. Figure 7(c) shows experimental results on BMS-Web View-1. FPMax* is faster than others and has a slight edge over SelPMiner for lower minimum support. FPMax* uses FP-array technique, which works very well, especially when the dataset is sparse. The FP-arrays save traversal time for all items and the next level FP-trees can be initialized directly. In this case, the time saved by omitting the first traversals is far greater than the time needed for accumulating counts in the associated FP-arrays. SelPMiner performs the same as FPMax* and PADS for low minimum support.

Figure 7(d) shows experimental results on BMS-Web View -2. Characteristics of all algorithms except Mafia are same for BMS-Web View-2 dataset. GenMax demonstrates the best performance of the six algorithms for low supports and is around 2-2.5 times faster than PADS. Figure 7(e) shows experimental results on Chainstore dataset, which is a large sparse dataset leading to a large number of transactions. GenMax especially works well for such datasets, i.e., sparse datasets, because of its TIDset representation.

The most striking performance improvements of SelPMiner were observed in the case of Webdocs, Chess, Pumsb, Pumsb*, Connect, Accidents and Retail datasets. This is because this dataset corresponds to cases where there are large numbers of very long patterns and SelPMiner successfully finds MFIs using faster recursive partitioning and longest support counting with better pruning. We observed that all experiments on all datasets for various minimum support show that our partition-based MFIs mining is indeed very effective when minimum support is very low.

\subsection{Memory consumption}

This section shows the peak memory consumption of all algorithms for various datasets. Both PADS and FPMax* use FP-trees as the major data structure. Thus, their memory usages are very similar. Their memory usage increases as the support threshold decreases, since they store the max-patterns already found in memory, and the number of such patterns increases as the support threshold decreases. GenMax consumes the maximum amount of memory for most of the datasets when minimum support is very low, 


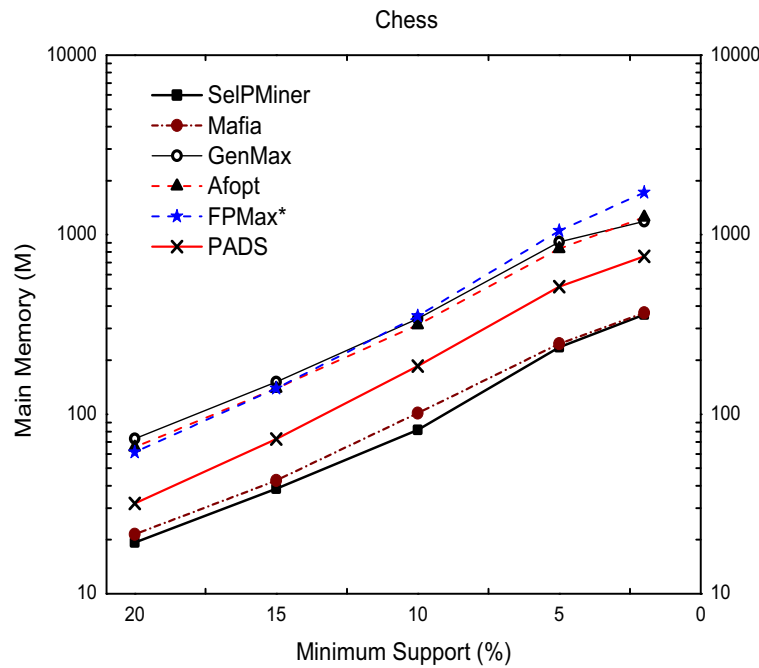

(a)

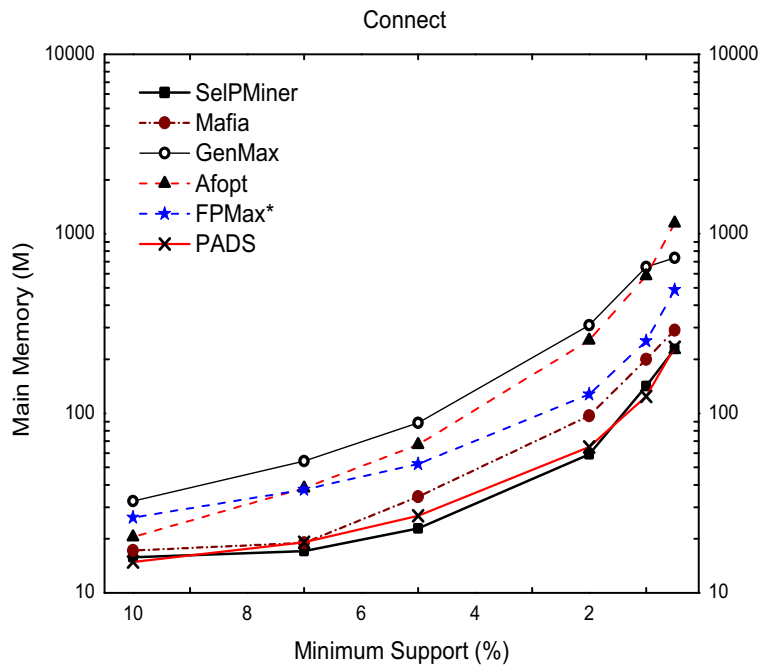

(c)

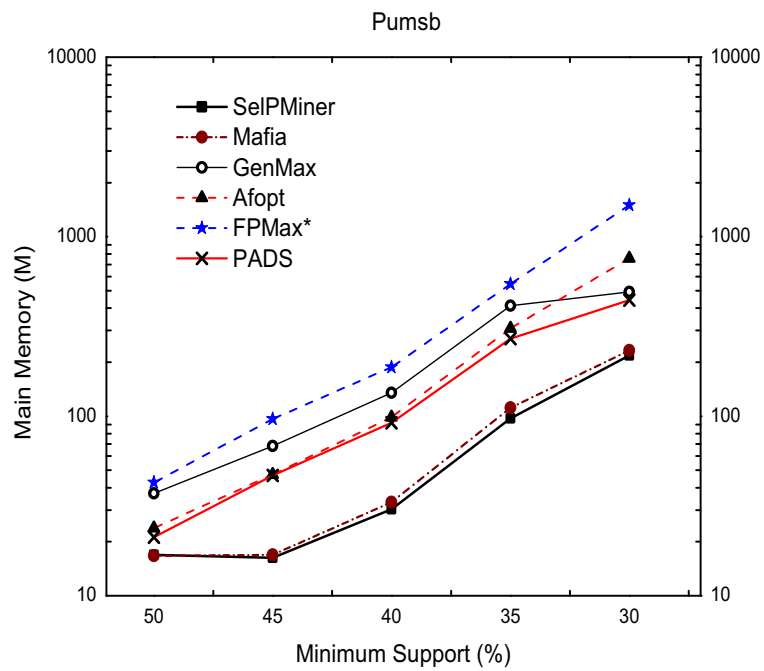

(b)

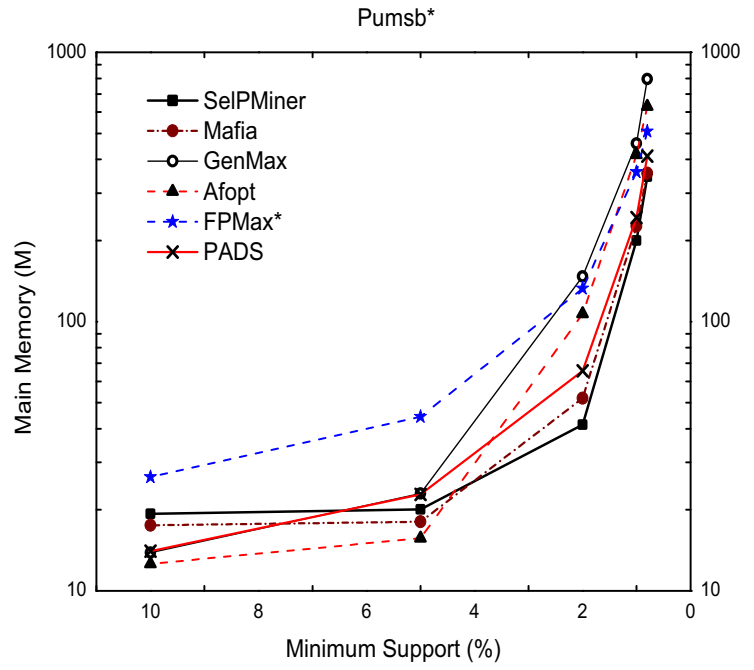

(d)

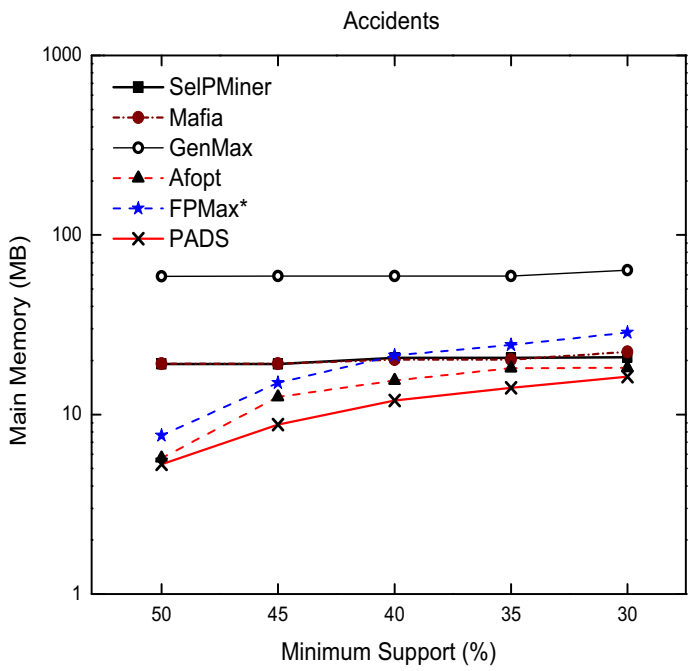

(e)

Figure 8. Memory consumption of mining MFIs on dense datasets. 
while SelPMiner was still able to run and produce output correctly.

The curves of GenMax and Afopt in figure 8(a) almost overlap the curve of FPMax*. It indicates that memory requirements for these algorithms are almost same. Memory consumption of SelPMiner is very less for dense dataset except for Accidents dataset, which can be seen from figure 8(a)-(d). This is because SelPMiner represents the dataset as RTPL, where for dense datasets the number of unique transactions will be less compared with number of unique transactions for sparse datasets. Mafia uses a large amount of memory in figure 8(c) and it consumes the least memory in figure 8(e) when the minimum support is very low. For most cases, GenMax consumes more memory than FPmax* for low minimum support. One observation from the two figures is that the memory consumption of FPmax* and GenMax increases exponentially when the minimum support becomes very low. From figure 8 we can observe that the increase happens because the algorithms have to store a large number of MFIs in memory, and the data structures such as MFItrees become very large. We can also see that when the memory needed by the algorithms increases rapidly, their runtime also increases very fast. The pair of figures 7(c) and 8(c) is a typical example. GenMax has the worst performance for Accidents dataset as it is a dense large dataset. Figure 9(a) shows the performance of all algorithms on Webdocs dataset, where SelPMiner consumes very less memory than those of other algorithms. GenMax uses the least memory in figure 9(b) for Retail dataset, when minimum support is low. Mafia increases exponentially with very low minimum support and this can be seen from figure $9(b)$.

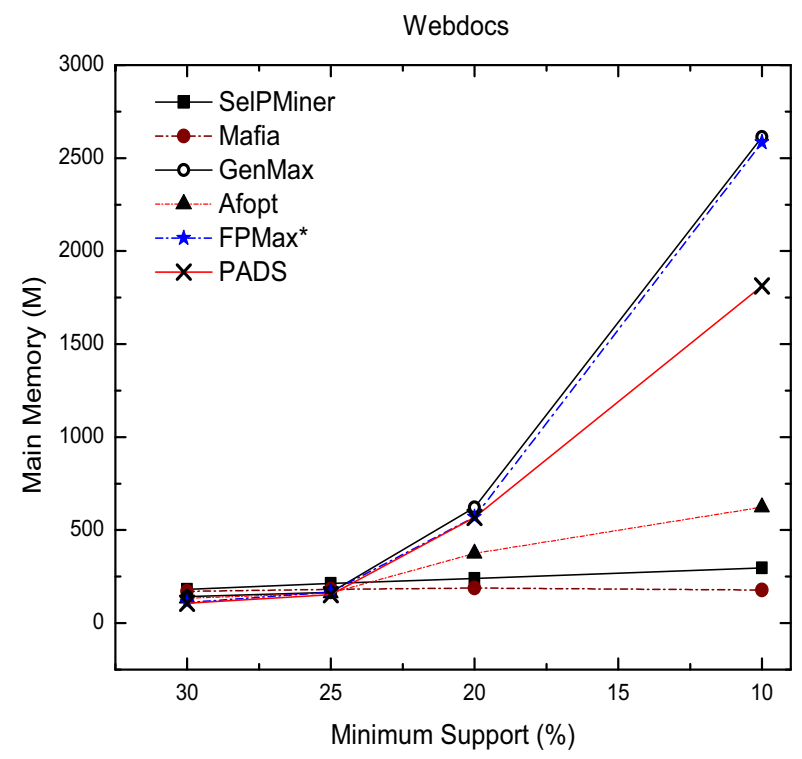

(a)

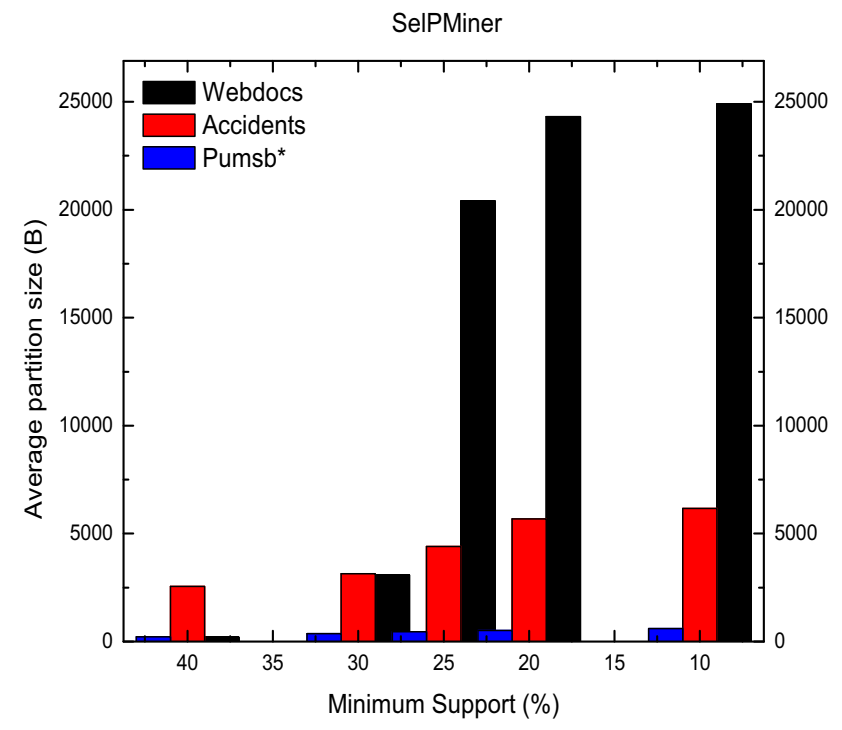

Figure 10. Average vs. minimum support.

Two observations from all figures are as follows: (1) the memory consumption of GenMax, Mafia and Afopt increases exponentially in many cases when minimum support becomes very low but SelPMiner gives the best performance and (2) the memory consumptions of FPMax* and PADS are larger than that of SelPMiner for dense datasets when minimum support becomes very low.

One bit represents one item in a data instance and hence the size of individual partition reduces exponentially during recursive partitioning as seen in figure 10 . Figure 10 shows average size of partitions during the selective recursive partitioning for Webdocs, Accidents and Pumsb* datasets.

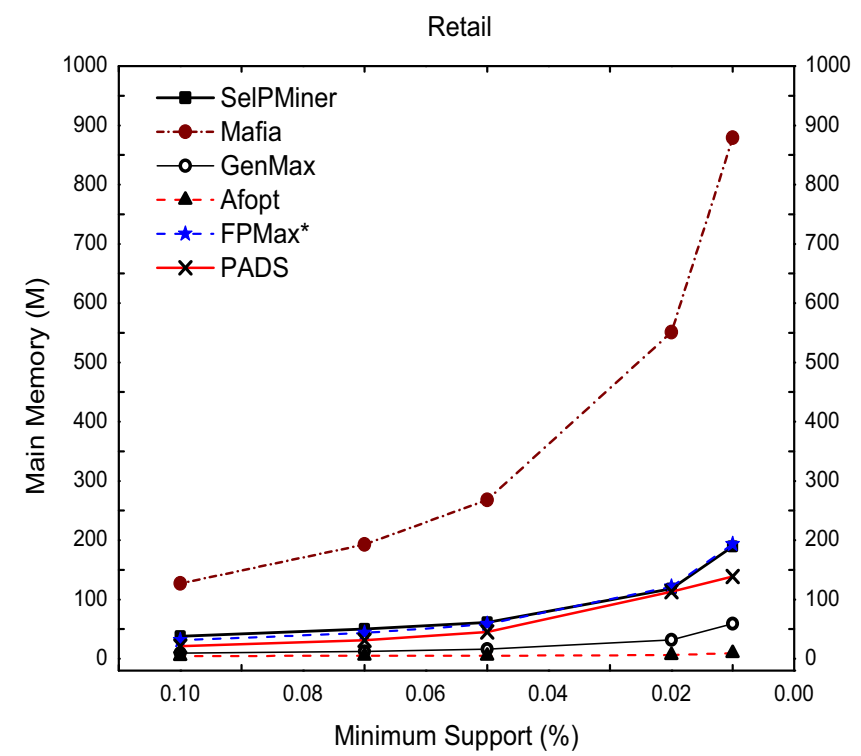

(b)

Figure 9. Memory consumption of mining MFIs on (a) Webdocs and (b) Retail dataset. 


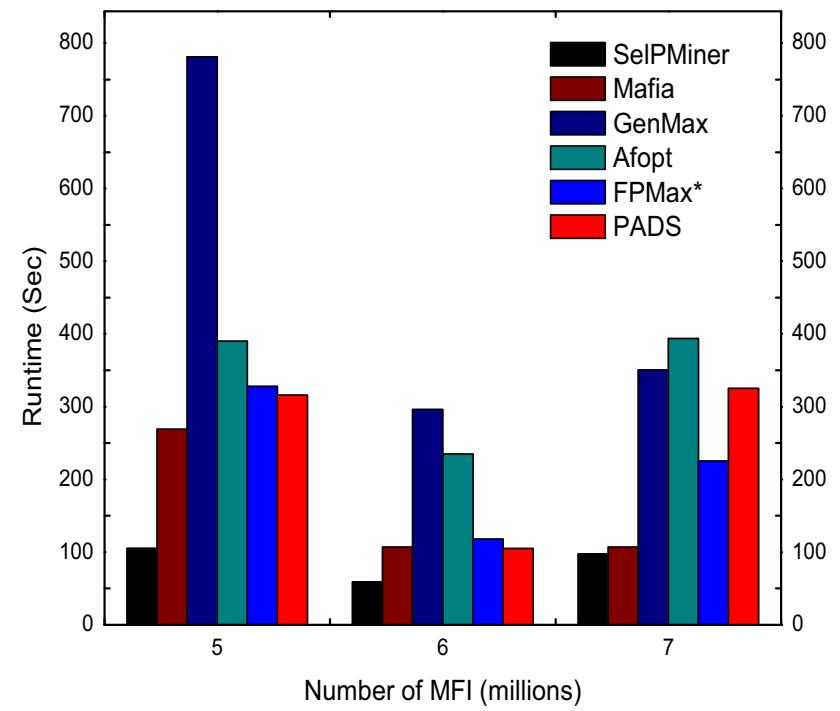

Figure 11. Runtime evaluation on MFI cardinality.

\subsection{Runtime evaluation on MFI cardinality}

We compare the total execution time for the different algorithms on MFI cardinality for Chess, Pumsb and Connect datasets. These datasets generate larger number of maximal FPs for very low minimum support, but also correspond to cases where there are large numbers of very long patterns (MFIs). Figure 11 shows runtime vs. generated number of maximal FPs. We evaluate Chess dataset with $\min \sup =2 \%$, Connect dataset with $\sup =0.5 \%$ and Pumsb dataset with sup $=30 \%$, which generates $7 \times 10^{6}$ MFIs, $6 \times 10^{6}$ MFIs and $5 \times 10^{6}$ MFIs, respectively. From figure 11 , we can say that for these three datasets, SelPMiner outperforms all other algorithms. The reason is most

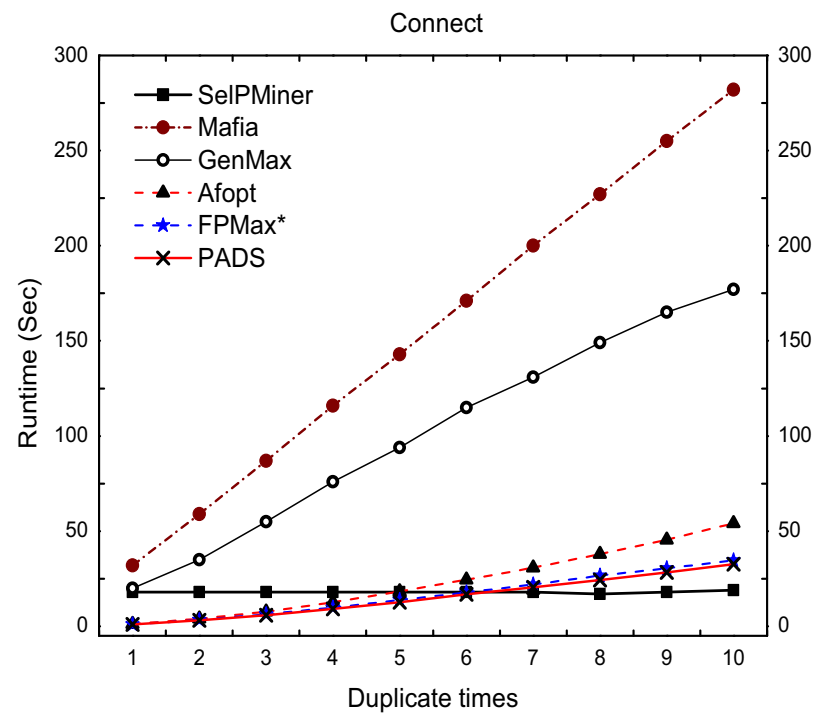

(a) likely due to the benefits of recursive partitioning, i.e., reducing dataset size, which results in efficient counting.

\subsection{Scalability of the algorithm}

To test the scalability of all six algorithms, we also run the programs on all duplicate datasets. Due to the lack of space, only the results for Connect dataset are shown here, while varying the number of transactions in the datasets from 2 to 10 times with $\min \sup =1.5 \%$.

Figure 12 shows the runtime and memory scalability of all algorithms on Connect duplicate dataset for mining MFIs. From figure 12(a) we can see that SelPMiner takes constant time. This is because the number of MFIs will not increase if we increase the number of transactions and keep other parameters, viz, number of items and distribution, unchanged; we can expect more similar transactions with increased count of bit-pattern in SelPMiner. The runtime of Mafia and GenMax algorithms increases rapidly, but none of the algorithms has an exponential runtime increase when the dataset size increases. PADS, FPMax* and Afopt algorithms take very less time for duplicate datasets but Afopt increases time when duplicate times increase. Figure shows that SelPMiner is a scalable algorithm. Figure 12(b) shows that PADS outperforms all algorithms. Mafia and GenMax consume much memory compared with others except Afopt for scaled dataset by duplicating. Memory consumption of SelPminer does not vary much.

\subsection{Algorithmic runtime analysis for partitioning}

To test the benefit of selective partitioning approach in SelPminer, we run the SelPMiner algorithm on four

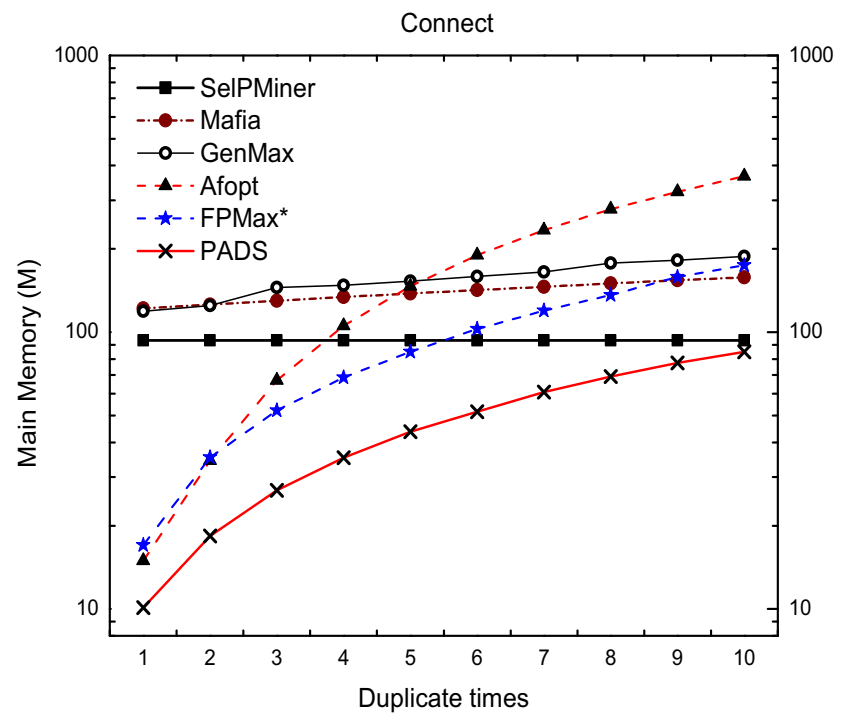

(b)

Figure 12. (a) Runtime and (b) memory consumption of mining MFIs on Connect duplicate dataset. 


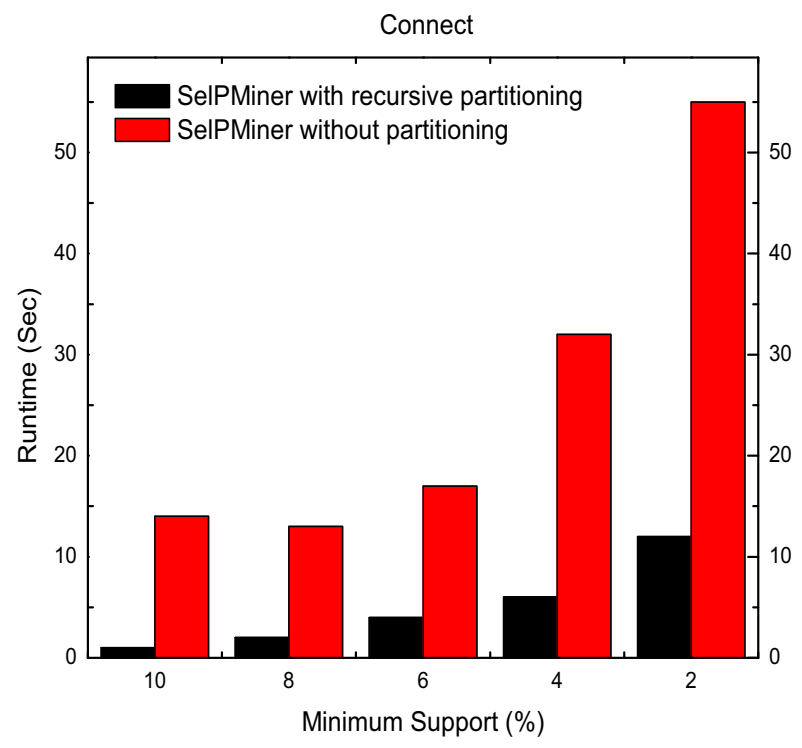

(a)

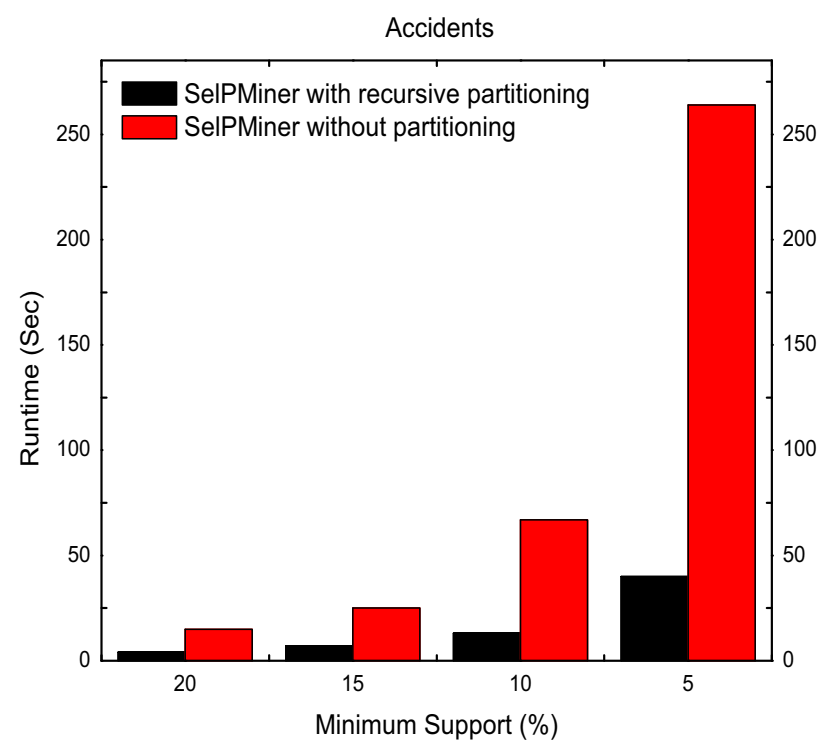

(c)

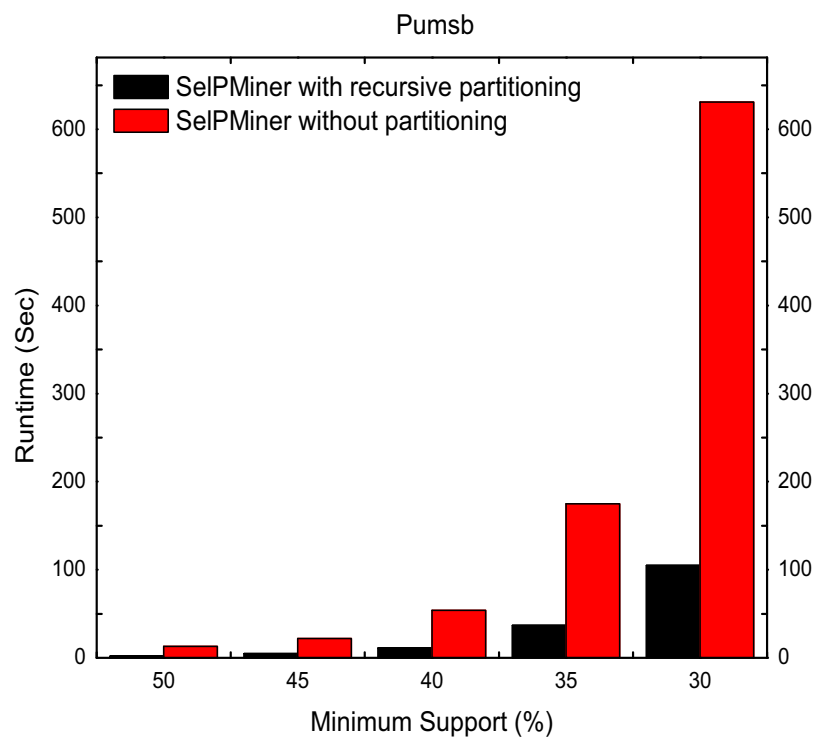

(b)

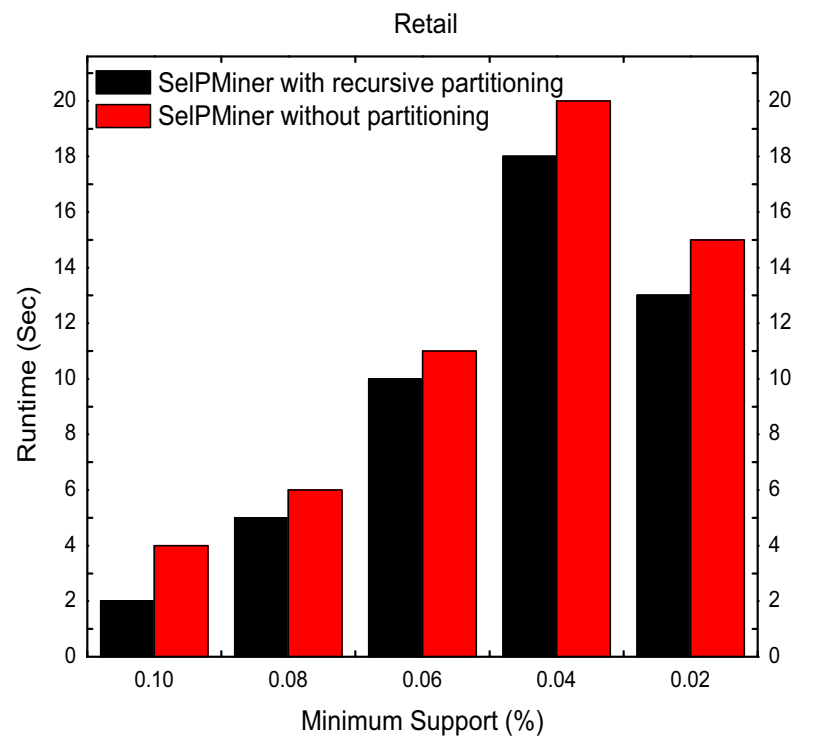

(d)

Figure 13. Runtime evaluation on partitioning.

datasets with partitioning statistics and with fixed partitioning. Figure 13(a)-(d) shows the results on Connect, Pumsb, Accidents and Retail datasets. All the figures show that partitioning approach saves 5 times CPU time compared with fixed partitioning.

\section{Conclusion}

We propose a way to store transactional data in a structure called 'partition'. We identify modified RTPL as its special case. While in operation, a partition compacts the data relevant to itemsets being investigated. The proposed MFI mining algorithm makes use of these partitions to significantly reduce the amount of time spent in counting supports of itemsets with very less amount of memory consumption. The search over partitions is optimized for faster retrieval of supersets of itemsets using unique binary search approach. This allows flexibility in queries over partitions. We have exploited this to increase the effectiveness of the FHUT pruning technique. The combined effect of faster support counting and better pruning resulted in the significant performance increase. We have also shown that at any time during its operation, the algorithm limits the number 
of tuples held by partitions to twice the number of transactions in the input database. For all datasets the proposed partition structure prunes the tree in its initial stage, so SelPMiner needs less amount of time and memory for computation. SelPMiner consumes very less memory than Mafia, Genmax, Afopt, FPmax* and PADS algorithms for all levels of minimum support.

The scalability of SelPMiner is also studied and found to be good. The algorithm is designed also in such a way that the result is independent of the order in which partitions are processed, making it suitable for distributed or parallel implementation. These partitions can be stored on disk and processed one by one.

Furthermore, we can try to parallelize the algorithm, to reduce the relative mining time and memory consumption on extremely large datasets. Additionally, it may be interesting to exploit partitions for finding occupancy-based FPs [5] specifically using modified RTPL. Because in real life, a database may dynamically change as the result of insertion, deletion and modification operations, for future work, we plan to extend the proposed algorithm to add the capability of incremental mining MFIs.

\section{References}

[1] Agrawal R and Srikant R 1994 Fast algorithms for mining association rules. In: Proceedings of the 20th International Conference on Very Large Databases, VLDB, vol. 1215, pp. 487-499

[2] Possas B, Ziviani N, Meira Jr W and Ribeiro-Neto B 2002 Set-based model: a new approach for information retrieval. In: Proceedings of the 25th Annual International ACM SIGIR Conference on Research and Development in Information Retrieval, pp. 230-237

[3] Sahoo J, Das A K and Goswami A 2015 An efficient approach for mining association rules from high utility itemsets. Expert Systems with Applications 42: 5754-5778

[4] Tseng V S, Wu C W, Fournier-Viger P and Philip S Y 2016 Efficient algorithms for mining top-k high utility itemsets. IEEE Transactions on Knowledge and Data Engineering 28: 54-67

[5] Zhang L, Luo P, Tang L, Chen E, Liu Q, Wang M and Xiong H 2015 Occupancy-based frequent pattern mining*. ACM Transactions on Knowledge Discovery from Data 10, article 14

[6] Konar P, Sil J and Chattopadhyay P 2015 Knowledge extraction using data mining for multi-class fault diagnosis of induction motor. Neurocomputing 166: 14-25

[7] Desai S, Pawar J and Bhattacharyya P 2015 Staged approach for grammatical gender identification of nouns using association rule mining and classification. Research in Computing Science 90: 359-371

[8] Kiran G V R, Shankar R and Pudi V 2010 Frequent itemset based hierarchical document clustering using wikipedia as external knowledge. In: Proceedings of the International Conference on Knowledge-Based and Intelligent Information and Engineering Systems, pp. 11-20
[9] Frawley W J, Piatetsky-Shapiro G and Matheus C J 1992 Knowledge discovery in databases: an overview. AI Magazine 13: 57-70

[10] Agrawal R, Imieliński T and Swami A 1993 Mining association rules between sets of items in large databases. In: Proceedings of the ACM SIGMOD International Conference on Management of Data, vol. 22, pp. 207-216

[11] Dimitrios G, Khardon R, Mannila H, Saluja S, Toivonen H and Sharma R S 2003 Discovering all most specific sentences. ACM Transactions on Database Systems 28: 140-174

[12] Yang G 2004 The complexity of mining maximal frequent itemsets and maximal frequent patterns. In: Proceedings of the 10th ACM SIGKDD International Conference on Knowledge Discovery and Data Mining, pp. 344-353

[13] Zaki M J 2000 Scalable algorithms for association mining. IEEE Transactions on Knowledge and Data Engineering 12: 372-390

[14] Sayal M and Scheuermann P 2001 Distributed web log mining using maximal large item sets. Knowledge Information Systems 3: 389-404

[15] Ordonez C, Ezquerra N and Santana C A 2006 Constraining and summarizing association rules in medical data. Knowledge Information Systems 9: 1-2

[16] Rigoutsos I and Floratos A 1998 Combinatorial pattern discovery in biological sequences: the TEIRESIAS algorithm. Bioinformatics 14: 55-67

[17] Lin D I and Kedem Z M 1998 Pincer-search: a new algorithm for discovering the maximum frequent set. In: Proceedings of the International Conference on Extending Database Technology. Berlin-Heidelberg: Springer, pp. 103-119

[18] Han J, Pei J, Yin Y and Mao R 2004 Mining frequent patterns without candidate generation: a frequent-pattern tree approach. Data Mining and Knowledge Discovery 8: 53-87

[19] Liu G, Lu H, Yu J X, Wang W and Xiao X 2003 AFOPT: an efficient implementation of pattern growth approach. In: Proceedings of FIMI

[20] Jiawei H and Pei J 2014 Pattern-growth methods. In: Aggarwal C and Han J (eds) Frequent Pattern Mining Workshop. Springer International Publishing, Cham, pp. $65-81$

[21] Grahne G and Zhu J 2003 High performance mining of maximal frequent itemsets. In: Proceedings of the 6th International Workshop on High Performance Data Mining

[22] Takeaki U, Kiyomi M and Arimura H 2004 LCM ver. 2: efficient mining algorithms for frequent/closed/maximal itemsets. In: Proceedings of FIMI, pp. 126

[23] Burdick D, Calimlim M, Flannick J, Gehrke J and Yiu T 2005 Mafia: a maximal frequent itemset algorithm. IEEE Transactions on Knowledge and Data Engineering 17: 1490-1504

[24] Roberto J and Bayardo J 1998 Efficiently mining long patterns from databases. ACM Sigmod Record 27: 85-93

[25] Gouda K and Zaki M J 2005 Genmax: an efficient algorithm for mining maximal frequent itemsets. Data Mining and Knowledge Discovery 11: 223-242

[26] Grahne G and Zhu J 2005 Fast algorithms for frequent itemset mining using fp-trees. IEEE Transactions on Knowledge and Data Engineering 17: 1347-1362

[27] Zeng X, Pei J, Wang K and Li J 2009 PADS: a simple yet effective pattern-aware dynamic search method for fast 
maximal frequent pattern mining. Knowledge and Information Systems 20: 375-391

[28] Jiawei H, Pei J and Kamber M 2011 Data mining: concepts and techniques, 3rd ed. USA: Morgan Kaufmann

[29] Rymon R 1992 Search through systematic set enumeration. In: Proceedings of the 3rd International Conference on Principles of Knowledge Representation and Reasoning, pp. 539-550

[30] Dhabu M and Deshpande P S 2012 Cardinality statistics based maximal frequent itemsets mining. In: Information Systems, Technology and Management Conference. BerlinHeidelberg: Springer, vol. 285, pp. 28-39

[31] Savasere A, Omiecinski E R and Navathe S B 1995 An efficient algorithm for mining association rules in large databases. Technical Report GIT-CC-95-04, Georgia Institute of Technology

[32] Xian W, Fan W, Peng J, Zhang K and Yu Y 2015 Iterative sampling based frequent itemset mining for big data. International Journal of Machine Learning and Cybernetics 6: $875-882$
[33] Anastasiu D C, Iverson J, Smith S and Karypis G 2014 Big data frequent pattern mining. In: Aggarwal C and Han J (eds) Frequent pattern mining. Springer International Publishing, Cham, pp. 225-259

[34] Shah D, Lakshmanan L V S, Ramamritham K and Sudarshan S 1999 Interestingness and pruning of mined patterns. In: Proceedings of the Data Mining and Knowledge Discovery Workshop, Philadelphia, PA, USA

[35] Zaki M J and Hsiao C J 2002 CHARM: an efficient algorithm for closed itemset mining. In: Proceedings of the 2002 SIAM International Conference on Data Mining, Society for Industrial and Applied Mathematics, pp. 457-473

[36] Pisharath J, Liu Y, Liao W K, Choudhary A and Memik G 2005 NU-MineBench version 2.0 dataset and technical report [Online]. Available: http://cucis.ece.northwestern.edu/pro jects/DMS/MineBench.html

[37] Chakaravarthy V T, Pandit V and Sabharwal Y 2009 Analysis of sampling techniques for association rule mining. In: Proceedings of the 12th International Conference on Database Theory, ACM, pp. 276-283 